\title{
The ample cone of moduli spaces of sheaves on the plane
}

\author{
Izzet Coskun and Jack Huizenga
}

\begin{abstract}
Let $\xi$ be the Chern character of a stable sheaf on $\mathbb{P}^{2}$. Assume either $\operatorname{rk}(\xi) \leqslant 6$ and that there are no strictly semistable sheaves with character $\xi$, or that $\operatorname{rk}(\xi)$ and $c_{1}(\xi)$ are coprime and the discriminant $\Delta(\xi)$ is sufficiently large. We use recent results of Bayer and Macrì on Bridgeland stability to compute the ample cone of the moduli space $M(\xi)$ of Gieseker-semistable sheaves on $\mathbb{P}^{2}$. We recover earlier results, such as those by Strømme and Yoshioka, as special cases.
\end{abstract}

\section{Introduction}

Let $\xi$ be the Chern character of a stable sheaf on $\mathbb{P}^{2}$. The moduli space $M(\xi)$ parameterizes $S$-equivalence classes of Gieseker-semistable sheaves with Chern character $\xi$. It is an irreducible, normal, factorial, projective variety [LP97]. In this paper, we determine the ample cone of $M(\xi)$ when either $\operatorname{rk}(\xi) \leqslant 6$ and there are no strictly semistable sheaves with character $\xi$, or $\operatorname{rk}(\xi)$ and $c_{1}(\xi)$ are coprime and the discriminant $\Delta(\xi)$ is sufficiently large.

The ample cone $\operatorname{Amp}(X)$ of a projective variety $X$ is the open convex cone in the Néron-Severi space spanned by the classes of ample divisors. It controls embeddings of $X$ into projective space and is among the most important invariants of $X$. Its closure, the nef cone $\operatorname{Nef}(X)$, is spanned by divisor classes that have nonnegative intersection with every curve on $X$ and is dual to the Mori cone of curves on $X$ under the intersection pairing (see [Laz04]). We now describe our results on $\operatorname{Amp}(M(\xi))$ in greater detail.

Let $\xi$ be an integral Chern character with rank $r>0$. We record such a character as a triple $(r, \mu, \Delta)$, where

$$
\mu=\frac{\mathrm{ch}_{1}}{r} \quad \text { and } \quad \Delta=\frac{1}{2} \mu^{2}-\frac{\mathrm{ch}_{2}}{r}
$$

are the slope and discriminant, respectively. We call the character $\xi$ (semi)stable if there exists a (semi)stable sheaf of character $\xi$. Drézet and Le Potier give an explicit curve $\delta(\mu)$ in the $(\mu, \Delta)$-plane such that the moduli space $M(\xi)$ is positive dimensional if and only if $\Delta \geqslant \delta(\mu)$

Received 31 October 2014, accepted in final form 8 June 2015.

2010 Mathematics Subject Classification 14J60 (primary), 14E30, 14J26, 14D20, $13 \mathrm{D} 02$ (secondary).

Keywords: moduli space of stable vector bundles, minimal model program, Bridgeland stability conditions, ample cone.

This journal is (C) Foundation Compositio Mathematica 2016. This article is distributed with Open Access under the terms of the Creative Commons Attribution Non-Commercial License, which permits non-commercial reuse, distribution, and reproduction in any medium, provided that the original work is properly cited. For commercial re-use, please contact the Foundation Compositio Mathematica.

During the preparation of this article the first author was partially supported by the NSF CAREER grant DMS-0950951535, and the second author was partially supported by a National Science Foundation Mathematical Sciences Postdoctoral Research Fellowship. 


\section{ThE AMPLE CONE OF MODUli SPACES OF SHEAVES ON THE PLANE}

[DLP85, LP97]. The vector bundles whose Chern characters satisfy $\Delta=\delta(\mu)$ are called heightzero bundles. Their moduli spaces have Picard group isomorphic to $\mathbb{Z}$. Hence, their ample cone is spanned by the positive generator and there is nothing further to discuss. Therefore, we will assume $\Delta>\delta(\mu)$, and say that $\xi$ has positive height.

There is a nondegenerate symmetric bilinear form on the $K$-group $K\left(\mathbb{P}^{2}\right)$ sending a pair of Chern characters $\xi, \zeta$ to the Euler characteristic $\chi\left(\xi^{*}, \zeta\right)$. When $\xi$ has positive height, the Picard group of the moduli space $M(\xi)$ is naturally identified with the orthogonal complement $\xi^{\perp}$ and is isomorphic to $\mathbb{Z} \oplus \mathbb{Z}$ [LP97]. Correspondingly, the Néron-Severi space is a 2dimensional vector space. In order to describe $\operatorname{Amp}(M(\xi))$, it suffices to specify its two extremal rays.

The moduli space $M(\xi)$ admits a surjective, birational morphism $j: M(\xi) \rightarrow M^{D U Y}(\xi)$ to the Donaldson-Uhlenbeck-Yau compactification $M^{D U Y}(\xi)$ of the moduli space of stable vector bundles (see [Li93] and [HL10]). As long as the locus of singular (that is, non-locally-free) sheaves in $M(\xi)$ is nonempty (see Theorem 2.2), the morphism $j$ is not an isomorphism and contracts curves (see Proposition 2.4). Consequently, the line bundle $\mathcal{L}_{1}$ defining $j$ is base-point-free but not ample (see [HL10]). It corresponds to a Chern character $u_{1} \in \xi^{\perp} \cong \operatorname{Pic} M(\xi)$ and spans an extremal ray of $\operatorname{Amp}(M(\xi))$. For all the characters $\xi$ that we will consider in this paper there are singular sheaves in $M(\xi)$, so one edge of $\operatorname{Amp}(M(\xi))$ is always spanned by $u_{1}$. We must compute the other edge of the cone, which we call the primary edge.

We now state our results. Let $\xi=(r, \mu, \Delta)$ be a stable Chern character. Let $\xi^{\prime}=\left(r^{\prime}, \mu^{\prime}, \Delta^{\prime}\right)$ be the stable Chern character satisfying the following defining properties:

- We have $0<r \leqslant r$ and $\mu^{\prime}<\mu$.

- Every rational number in the interval $\left(\mu^{\prime}, \mu\right)$ has denominator greater than $r$.

- The discriminant of any stable bundle of slope $\mu^{\prime}$ and rank at most $r$ is at least $\Delta^{\prime}$.

- The minimal rank of a stable Chern character with slope $\mu^{\prime}$ and discriminant $\Delta^{\prime}$ is $r^{\prime}$.

The character $\xi^{\prime}$ is easily computed using Drézet and Le Potier's classification of stable bundles.

Theorem 1.1. Let $\xi=(r, \mu, \Delta)$ be a positive-height Chern character such that $r$ and $c_{1}$ are coprime. Suppose that $\Delta$ is sufficiently large, depending on $r$ and $\mu$. The cone $\operatorname{Amp}(M(\xi))$ is spanned by $u_{1}$ and a negative-rank character in $\left(\xi^{\prime}\right)^{\perp}$.

The required lower bound on $\Delta$ can be made explicit; see Remark 8.7. Our second result computes the ample cone of small-rank moduli spaces.

Theorem 1.2. Let $\xi=(r, \mu, \Delta)$ be a positive-height Chern character with $r \leqslant 6$.

(i) If $\xi$ is not a twist of $\left(6, \frac{1}{3}, \frac{13}{18}\right)$ and there are no strictly semistable sheaves with character $\xi$, then $\operatorname{Amp}(M(\xi))$ is spanned by $u_{1}$ and a negative-rank character in $\left(\xi^{\prime}\right)^{\perp}$.

(ii) If $\xi=\left(6, \frac{1}{3}, \frac{13}{18}\right)$, then $\operatorname{Amp}(M(\xi))$ is spanned by $u_{1}$ and a negative-rank character in $\left(\operatorname{ch} \mathcal{O}_{\mathbb{P}^{2}}\right)^{\perp}$.

The new ingredient that allows us to calculate $\operatorname{Amp}(M(\xi))$ is Bridgeland stability. Bridgeland [Bri07, Bri08] and Arcara and Bertram [AB13] construct Bridgeland stability conditions on the bounded derived category of coherent sheaves on a projective surface. On $\mathbb{P}^{2}$, these stability conditions $\sigma_{s, t}=\left(\mathcal{A}_{s}, Z_{s, t}\right)$ are parameterized by a half-plane $H:=\{(s, t) \mid s, t \in \mathbb{R}, t>0\}$ (see [ABCH13] and Section 2). Given a Chern character $\xi$, the half-plane $H$ admits a finite 


\section{Coskun And J. Huizenga}

wall-and-chamber decomposition, where in each chamber the collection of $\sigma_{s, t}$-semistable objects with Chern character $\xi$ remains constant. These walls are disjoint and consist of a vertical line $s=\mu$ and nested semicircles with center along $t=0$ [ABCH13]. In particular, there is a largest semicircular wall $W_{\max }$ to the left of the vertical wall. We will call this wall the Gieseker wall. Outside this wall, the moduli space $M_{\sigma_{s, t}}(\xi)$ of $\sigma_{s, t}$-semistable objects is isomorphic to the Gieseker moduli space $M(\xi)$ [ABCH13].

Let $\sigma_{0}$ be a stability condition on the Gieseker wall for $M(\xi)$. Bayer and Macrì [BM14b] construct a nef divisor $\ell_{\sigma_{0}}$ on $M(\xi)$ corresponding to $\sigma_{0}$. They also characterize the curves $C$ in $M(\xi)$ which have intersection number 0 with $\ell_{\sigma_{0}}$, as follows: $\ell_{\sigma_{0}} \cdot C=0$ if and only if two general sheaves parameterized by $C$ are $S$-equivalent with respect to $\sigma_{0}$ (that is, their Jordan-Hölder factors with respect to the stability condition $\sigma_{0}$ are the same). The divisor $\ell_{\sigma_{0}}$ is therefore an extremal nef divisor if and only if such a curve in $M(\xi)$ exists. This divisor can also be constructed via the geometric invariant theory (GIT) methods of Li and Zhao [LZ13].

In light of the results of Bayer and Macrì, our proofs of Theorems 1.1 and 1.2 amount to the computation of the Gieseker wall. For simplicity, we describe our approach to proving Theorem 1.1; the basic strategy for the proof of Theorem 1.2 is similar.

Theorem 1.3. Let $\xi$ be as in Theorem 1.1. The Gieseker wall for $M(\xi)$ is the wall $W\left(\xi^{\prime}, \xi\right)$ where $\xi$ and $\xi^{\prime}$ have the same Bridgeland slope.

There are two parts to the proof of this theorem. First, we show that $W_{\max }$ is no larger than $W\left(\xi^{\prime}, \xi\right)$. This is a numerical computation based on Bridgeland stability. The key technical result (Theorem 8.2) is that if a wall is larger than $W\left(\xi^{\prime}, \xi\right)$, then the rank of a destabilizing subobject corresponding to the wall is at most $\operatorname{rk}(\xi)$. We then find that the extremality properties defining $\xi^{\prime}$ guarantee that $W\left(\xi^{\prime}, \xi\right)$ is at least as large as any wall for $M(\xi)$ (Theorem 8.6).

In the other direction, we must show that $W\left(\xi^{\prime}, \xi\right)$ is an actual wall for $M(\xi)$. Define a character $\xi^{\prime \prime}=\xi-\xi^{\prime}$. Our next theorem produces a sheaf $E \in M(\xi)$ which is destabilized along $W\left(\xi^{\prime}, \xi\right)$.

Theorem 1.4. Let $\xi$ be as in Theorem 1.1. Fix general sheaves $F \in M\left(\xi^{\prime}\right)$ and $Q \in M\left(\xi^{\prime \prime}\right)$. Then the general sheaf $E$ given by an extension

$$
0 \rightarrow F \rightarrow E \rightarrow Q \rightarrow 0
$$

is Gieseker stable. Furthermore, we obtain curves in $M(\xi)$ by varying the extension class.

If $E$ is a Gieseker-stable extension as in the theorem, then $E$ is strictly semistable with respect to a stability condition $\sigma_{0}$ on $W\left(\xi^{\prime}, \xi\right)$, and not semistable with respect to a stability condition below $W\left(\xi^{\prime}, \xi\right)$. Thus $W\left(\xi^{\prime}, \xi\right)$ is an actual wall for $M(\xi)$, and it is the Gieseker wall. Any two Gieseker-stable extensions of $Q$ by $F$ are $S$-equivalent with respect to $\sigma_{0}$, so any curve $C$ in $M(\xi)$ obtained by varying the extension class satisfies $\ell_{\sigma_{0}} \cdot C=0$. Therefore, $\ell_{\sigma_{0}}$ spans an edge of the ample cone. Dually, $C$ spans an edge of the Mori cone of curves.

The natural analogs of Theorems 1.3 and 1.4 are almost true when instead $\operatorname{rk}(\xi) \leqslant 6$ as in Theorem 1.2; some minor adjustments to the statements need to be made for certain smalldiscriminant cases. See Theorems 7.6, 8.9, and 8.10, and Propositions 7.7 and 7.8 for precise statements. As the rank increases beyond 6, these exceptions become more common, and many more ad hoc arguments are required when using current techniques.

Bridgeland stability conditions were effectively used to study the birational geometry of Hilbert schemes of points on $\mathbb{P}^{2}$ in $[\mathrm{ABCH} 13]$ and moduli spaces of rank 0 semistable sheaves 


\section{ThE AMPLE CONE OF MODUli SPACES OF SHEAVES ON THE PLANE}

in [Woo13]. The ample cone of $M(\xi)$ was computed earlier for some special Chern characters. The ample cone of the Hilbert scheme of points on $\mathbb{P}^{2}$ was computed in [LQZ03] (see also [ABCH13, Ohk10]). Strømme computed $\operatorname{Amp}(M(\xi))$ when the rank of $\xi$ is 2 and either $c_{1}$ or $c_{2}-\frac{1}{4} c_{1}^{2}$ is odd [Str84]. Similarly, when the slope is $1 / r$, Yoshioka [Yos03] computed the ample cone of $M(\xi)$ and described the first flip. Our results contain these as special cases. Bridgeland stability has also been effectively used to compute ample cones of moduli spaces of sheaves on other surfaces. For example, see [AB13, BM14b, BM14a, MYY11, MYY14] for K3 surfaces, [MM13, Yos12, YY14] for abelian surfaces, [Nue14] for Enriques surfaces, and [BC13] for the Hilbert scheme of points on Hirzebruch surfaces and del Pezzo surfaces.

\section{Organization of the paper}

In Section 2, we introduce the necessary background on $M(\xi)$ and Bridgeland stability conditions on $\mathbb{P}^{2}$. In Sections 3 and 4 , we study the stability of extensions of sheaves and prove the first statement in Theorem 1.4. In Sections 5 and 6, we prove the analog of the first assertion in Theorem 1.4 for $\operatorname{rk}(\xi) \leqslant 6$. In Section 7, we complete the proof of Theorem 1.4 (and its smallrank analog) by constructing the desired curves of extensions. Finally, in Section 8, we compute the Gieseker wall, completing the proofs of Theorems 1.1 and 1.2.

\section{Preliminaries}

In this section, we recall basic facts concerning the classification of stable vector bundles on $\mathbb{P}^{2}$ and Bridgeland stability.

\subsection{Stable sheaves on $\mathbb{P}^{2}$}

Let $\xi$ be the Chern character of a (semi)stable sheaf on $\mathbb{P}^{2}$. We will call such characters (semi)stable characters. The classification of stable characters on $\mathbb{P}^{2}$ due to Drézet and Le Potier is best stated in terms of the slope $\mu$ and the discriminant $\Delta$. Let

$$
P(m)=\frac{1}{2}\left(m^{2}+3 m+2\right)
$$

denote the Hilbert polynomial of $\mathcal{O}_{\mathbb{P}^{2}}$. The Euler characteristic of the pair $(E, F)$ is defined by

$$
\chi(E, F)=\sum_{i=0}^{2}(-1)^{i} \operatorname{ext}^{i}(E, F),
$$

where $\operatorname{ext}^{i}(E, F)=\operatorname{dim}_{\operatorname{Ext}^{i}}(E, F)$. In terms of these invariants, the Riemann-Roch formula reads

$$
\chi(E, F)=\operatorname{rk}(E) \operatorname{rk}(F)(P(\mu(F)-\mu(E))-\Delta(E)-\Delta(F)) .
$$

An exceptional bundle $E$ on $\mathbb{P}^{2}$ is a stable bundle such that $\operatorname{Ext}^{1}(E, E)=0$. The exceptional bundles are rigid; their moduli spaces consist of a single reduced point [LP97, Corollary 16.1.5]. They are the stable bundles $E$ on $\mathbb{P}^{2}$ with $\Delta(E)<\frac{1}{2}$ [LP97, Proposition 16.1.1]. Examples of exceptional bundles include the line bundles $\mathcal{O}_{\mathbb{P}^{2}}(n)$ and the tangent bundle $T_{\mathbb{P}^{2}}$. All exceptional bundles can be obtained from line bundles via a sequence of mutations [Dre86]. An exceptional slope $\alpha \in \mathbb{Q}$ is the slope of an exceptional bundle. If $\alpha$ is an exceptional slope, there is a unique exceptional bundle $E_{\alpha}$ with slope $\alpha$. The rank of the exceptional bundle is the smallest positive 


\section{Coskun And J. Huizenga}

integer $r_{\alpha}$ such that $r_{\alpha} \alpha$ is an integer. The discriminant $\Delta_{\alpha}$ is then given by

$$
\Delta_{\alpha}=\frac{1}{2}\left(1-\frac{1}{r_{\alpha}^{2}}\right)
$$

The set $\mathscr{E}$ of exceptional slopes is well understood (see [DLP85] and [CHW15]).

The classification of positive-dimensional moduli spaces of stable vector bundles on $\mathbb{P}^{2}$ is expressed in terms of a fractal-like curve $\delta$ in the $(\mu, \Delta)$-plane. For each exceptional slope $\alpha \in \mathscr{E}$, there is an interval $I_{\alpha}=\left(\alpha-x_{\alpha}, \alpha+x_{\alpha}\right)$ with

$$
x_{\alpha}=\frac{3-\sqrt{5+8 \Delta_{\alpha}}}{2}
$$

such that the function $\delta(\mu)$ is defined on $I_{\alpha}$ by the function

$$
\delta(\mu)=P(-|\mu-\alpha|)-\Delta_{\alpha} \quad \text { if } \quad \alpha-x_{\alpha}<\mu<\alpha+x_{\alpha} .
$$

The graph of $\delta(\mu)$ is an increasing concave up parabola on the interval $\left[\alpha-x_{\alpha}, \alpha\right]$ and a decreasing concave up parabola on the interval $\left[\alpha, \alpha+x_{\alpha}\right]$. The function $\delta$ is invariant under translation by integers. The main classification theorem of Drézet and Le Potier is as follows.

Theorem 2.1 ([DLP85, LP97]). There exists a positive-dimensional moduli space of Giesekersemistable sheaves $M(\xi)$ with integral Chern character $\xi$ if and only if $\Delta \geqslant \delta(\mu)$. In this case, $M(\xi)$ is a normal, irreducible, factorial projective variety of dimension $r^{2}(2 \Delta-1)+1$.

\subsection{Singular sheaves on $\mathbb{P}^{2}$}

To study one extremal edge of the ample cone, we need to understand the locus of singular sheaves in $M(\xi)$. The following theorem, which is likely well known to experts, characterizes the Chern characters where the locus of singular sheaves in $M(\xi)$ is nonempty. We include a proof for lack of a convenient reference.

Theorem 2.2. Let $\xi=(r, \mu, \Delta)$ be an integral Chern character with $r>0$ and $\Delta \geqslant \delta(\mu)$. The locus of singular sheaves in $M(\xi)$ is empty if and only if $\Delta-\delta(\mu)<1 / r$ and $\mu$ is not an exceptional slope.

Proof. Let $F$ be a singular sheaf in $M(\xi)$. Then $F^{* *}$ is a $\mu$-semistable, locally free sheaf [HL10, Section 8] with invariants

$$
\operatorname{rk}\left(F^{* *}\right)=r, \quad \mu\left(F^{* *}\right)=\mu, \quad \text { and } \quad \Delta\left(F^{*}\right) \leqslant \Delta(F)-\frac{1}{r} .
$$

Since the set $\mathbb{R}-\cup_{\alpha \in \mathscr{E}} I_{\alpha}$ does not contain any rational numbers [DLP85], [CHW15, Theorem 4.1], $\mu$ is an element of $I_{\alpha}$ for some exceptional slope $\alpha$. Let $E_{\alpha}$ with invariants $\left(r_{\alpha}, \alpha, \Delta_{\alpha}\right)$ be the corresponding exceptional bundle.

If $\Delta-\delta(\mu)<1 / r$ and $F$ is a singular sheaf in $M(\xi)$, then $\Delta\left(F^{* *}\right)<\delta(\mu)$. If $\alpha>\mu$, then $\chi\left(E_{\alpha}, F^{* *}\right)>0$. Since $\operatorname{Ext}^{2}\left(E_{\alpha}, F^{* *}\right)$ vanishes by Serre duality, we have $\operatorname{hom}\left(E_{\alpha}, F^{* *}\right)>0$. Similarly, if $\alpha<\mu$, then $\operatorname{hom}\left(F^{* *}, E_{\alpha}\right)>0$. In either case, these homomorphisms violate the $\mu$-semistability of $F^{* *}$, leading to a contradiction. Therefore, if $\Delta-\delta(\mu)<1 / r$ and $\mu$ is not an exceptional slope, then the locus of singular sheaves in $M(\xi)$ is empty.

To prove the converse, we construct singular sheaves using elementary modifications. If $\Delta-$ $\delta(\mu) \geqslant 1 / r$, then $\zeta=(r, \mu, \Delta-1 / r)$ is a stable Chern character. Let $G$ be a $\mu$-stable bundle in $M(\zeta)$, which exists by [DLP85, Corollary 4.12]. Choose a point $p \in \mathbb{P}^{2}$, and let $G \rightarrow \mathcal{O}_{p}$ be a 


\section{The AMPle CONE OF MODUli SPACES OF SHEAVES ON THE Plane}

general surjection. Then the kernel sheaf $F$ defined by

$$
0 \rightarrow F \rightarrow G \rightarrow \mathcal{O}_{p} \rightarrow 0
$$

is a $\mu$-stable, singular sheaf with Chern character $\xi$ (see Section 5 for more details on elementary modifications).

We are reduced to showing that if $\mu=\alpha$ and $\Delta-\delta(\alpha)<1 / r$, then the locus of singular sheaves in $M(\xi)$ is nonempty. Since $c_{1}\left(E_{\alpha}\right)$ and $r_{\alpha}$ are coprime, the rank of any bundle with slope $\alpha$ is a multiple of $r_{\alpha}$. Write

$$
r=k r_{\alpha}^{2}+m r_{\alpha}, \quad 0 \leqslant k, 0<m \leqslant r_{\alpha} .
$$

By integrality, there exists an integer $N$ such that $\Delta-N / r=\Delta_{\alpha}$. Our choice of $k$ implies

$$
\Delta=\Delta_{\alpha}+\frac{k+1}{r} \text {. }
$$

First, assume $k=0$. If $r^{\prime}<r$, then $\Delta_{\alpha}+1 / r^{\prime}>\Delta_{\alpha}+1 / r$. Consequently, the only Giesekersemistable sheaves of character $\left(r^{\prime}, \mu, \Delta^{\prime}\right)$ with $r^{\prime}<r$ and $\Delta^{\prime}<\Delta$ are semiexceptional sheaves $E_{\alpha}^{\oplus \ell}$ with $\ell<m$. Let $F$ be a general elementary modification of the form

$$
0 \rightarrow F \rightarrow E_{\alpha}^{\oplus m} \rightarrow \mathcal{O}_{p} \rightarrow 0 .
$$

Then $F$ is a $\mu$-semistable singular sheaf with Chern character $\xi$. If $F$ were not Gieseker semistable, then it would admit an injective map $\phi: E_{\alpha} \rightarrow F$. By Lemma 2.3 below, for a general surjection $\psi: E_{\alpha}^{\oplus m} \rightarrow \mathcal{O}_{p}$, there does not exist an injection $E_{\alpha} \rightarrow E_{\alpha}^{\oplus m}$ which maps to 0 under $\psi$. Composing $\phi$ with the maps in the exact sequence defining $F$, we get a contradiction. We conclude that $F$ is Gieseker semistable. This constructs singular sheaves when $k=0$.

Next, assume $k>0$. If $m=r_{\alpha}$, then we can construct a singular sheaf in $M(\xi)$ as a $(k+1)$ fold direct sum of a semistable singular sheaf constructed in the case $k=0, m=r_{\alpha}$. Hence, we may assume $m<r_{\alpha}$. Let $G$ be a $\mu$-stable vector bundle with Chern character

$$
\zeta=\left(k r_{\alpha}^{2}, \alpha, \delta(\alpha)=\Delta_{\alpha}+\frac{1}{r_{\alpha}^{2}}\right)
$$

Note that $(\mu(\zeta), \Delta(\zeta))$ lies on the curve $\delta$, hence $\chi\left(E_{\alpha}, G\right)=\chi\left(G, E_{\alpha}\right)=0$. Every locally free sheaf in $M(\zeta)$ has a two-step resolution in terms of exceptional bundles orthogonal to $E_{\alpha}$ [DLP85]. Consequently, $\operatorname{hom}\left(G, E_{\alpha}\right)=0$. We also have $\operatorname{hom}\left(E_{\alpha}, G\right)=0$ by stability.

Let $\phi: E_{\alpha}^{\oplus m} \oplus G \rightarrow \mathcal{O}_{p}$ be a general surjection, and let $F$ be defined as the corresponding elementary modification

$$
0 \rightarrow F \rightarrow E_{\alpha}^{\oplus m} \oplus G \rightarrow \mathcal{O}_{p} \rightarrow 0
$$

We first check that the Chern character of $F$ is $\xi$. Clearly, $\operatorname{rk}(F)=r$ and $\mu(F)=\alpha$. The discriminant equals

$$
\Delta(F)=\frac{1}{r}\left(m r_{\alpha} \Delta_{\alpha}+k r_{\alpha}^{2}\left(\Delta_{\alpha}+\frac{1}{r_{\alpha}^{2}}\right)\right)+\frac{1}{r}=\Delta_{\alpha}+\frac{k+1}{r}=\Delta .
$$

Hence, $F$ is a singular sheaf with the correct invariants. It remains to check that it is Gieseker semistable. Note that $F$ is at least $\mu$-semistable.

Suppose that $\psi: U \rightarrow F$ is an injection from a Gieseker-stable sheaf $U$ that destabilizes $F$. Since $F$ is $\mu$-semistable, $\mu(U)=\alpha$ and $\Delta(U)<\Delta$. Then we claim that either $U=E_{\alpha}$ or $\operatorname{rk}(U)>m r_{\alpha}$. Suppose $U \neq E_{\alpha}$ and $\operatorname{rk}(U)=s r_{\alpha}$. Then

$$
\Delta=\Delta_{\alpha}+\frac{k+1}{r}>\Delta(U) \geqslant \Delta_{\alpha}+\frac{1}{s r_{\alpha}} \text {. }
$$




\section{Coskun And J. Huizenga}

Hence,

$$
s>\frac{k r_{\alpha}+m}{k+1}>\frac{k m+m}{k+1}=m .
$$

If $U \neq E_{\alpha}$, composing $\psi$ with the inclusion to $E_{\alpha}^{\oplus m} \oplus G$ gives an injection $\psi^{\prime}: U \rightarrow E_{\alpha}^{\oplus m} \oplus G$. Since $\operatorname{rk}(U)>m r_{\alpha}$, the projection to $G$ cannot be 0 . Hence, we get a nonzero map $\vartheta: U \rightarrow G$. Let $V=\operatorname{Im} \vartheta$. We have $\operatorname{rk} V=\operatorname{rk} G$ by the $\mu$-stability of $G$. We claim that $\vartheta$ is in fact surjective. The quotient $G / V$ is 0 -dimensional by stability, and, if it is nonzero, then

$$
\Delta(U)<\Delta<\delta(\alpha)+\frac{1}{r}<\delta(\alpha)+\frac{1}{k r_{\alpha}^{2}} \leqslant \Delta(V) .
$$

This violates the stability of $U$, so $V=G$ and $\vartheta$ is surjective. If $\operatorname{rk}(U)=\operatorname{rk}(G)$, then $U \cong G$ and $\psi^{\prime}$ maps $U$ isomorphically onto $G \subset E_{\alpha}^{\oplus m} \oplus G$. A general hyperplane in the fiber $\left(E_{\alpha}^{\oplus m} \oplus G\right)_{p}$ is transverse to $G_{p}$, so this contradicts the fact that $\phi \circ \psi^{\prime}=0$ and $\phi$ is general. Suppose $\operatorname{rk}(U)>\operatorname{rk}(G)$, and write

$$
\operatorname{rk}(U)=k r_{\alpha}^{2}+n r_{\alpha}
$$

with $0<n<m$. Then we find

$$
\Delta_{\alpha}+\frac{k+1}{r}=\Delta>\Delta(U) \geqslant \Delta_{\alpha}+\frac{k+1}{\operatorname{rk}(U)},
$$

contradicting $\operatorname{rk}(U)<r$. We conclude that if $U \neq E_{\alpha}$, then $U$ cannot destabilize $F$.

On the other hand, if $U=E_{\alpha}$, then by the semistability of $G$ the composition of $\psi^{\prime}$ with the projection to $G$ must be 0 . A general hyperplane in the fiber $\left(E_{\alpha}^{\oplus m} \oplus G\right)_{p}$ intersects $\left(E_{\alpha}^{\oplus m}\right)_{p}$ in a hyperplane $H \subset\left(E_{\alpha}^{\oplus m}\right)_{p}$. Since $m \leqslant r_{\alpha}$, Lemma 2.3 shows that the composition of $\psi^{\prime}$ with $\phi$ is nonzero, giving a contradiction. We conclude that $F$ is Gieseker semistable.

Lemma 2.3. Let $E_{\alpha}$ be an exceptional bundle of rank $r_{\alpha}$. Let $H$ be a general codimension $c$ subspace of the fiber of $E_{\alpha}^{\oplus m}$ over a point $p$. Then there exists an injection $\phi: E_{\alpha} \rightarrow E_{\alpha}^{\oplus m}$ such that $\phi_{p}\left(E_{\alpha}\right) \subset H$ if and only if $c r_{\alpha} \leqslant m-1$.

Proof. For simplicity set $E=E_{\alpha}$ and $r=r_{\alpha}$. Let $S$ denote the Segre embedding of $\mathbb{P}^{r-1} \times \mathbb{P}^{m-1}$ in $\mathbb{P}^{r m-1}$. Let $q_{1}$ and $q_{2}$ denote the two projections from $S$ to $\mathbb{P}^{r-1}$ and $\mathbb{P}^{m-1}$, respectively. We will call a linear $\mathbb{P}^{r-1}$ in $S$ contracted by $q_{2}$ a $\mathbb{P}^{r-1}$ fiber.

Let $\phi: E \rightarrow E^{\oplus m}$ be an injection. Composing $\phi$ with the $m$ projections, we get $m$ morphisms $E \rightarrow E$. Since $E$ is simple, the resulting maps are all homotheties. Let $M=\left(\begin{array}{lllll}\lambda_{1} I & \lambda_{2} I & \ldots & \lambda_{m} I\end{array}\right)$ be the $r \times r m$ matrix, where $I$ is the $r \times r$ identity matrix and the $\lambda_{i}$ are scalars. Let $\vec{x}=$ $\left(x_{1}, \ldots, x_{r}\right)^{T}$. Hence, $\phi_{p}(E)$ has the form

$$
M \vec{x}=\left(\lambda_{1} x_{1}, \lambda_{1} x_{2}, \ldots, \lambda_{1} x_{r}, \ldots, \lambda_{m} x_{1}, \ldots, \lambda_{m} x_{r}\right)^{T} .
$$

If we projectivize, we see that the fibers $\mathbb{P}\left(\phi_{p}(E)\right)$ are $\mathbb{P}^{r-1}$ fibers contained in the Segre embedding of $\mathbb{P}^{r-1} \times \mathbb{P}^{m-1}$ in $\mathbb{P}\left(\left(E^{\oplus m}\right)_{p}\right)$. Conversely, every $\mathbb{P}^{r-1}$ fiber in $S$ is obtained by fixing a point $\left(\lambda_{1}, \ldots, \lambda_{m}\right) \in \mathbb{P}^{m-1}$ and, hence, is the fiber of an injection $E \rightarrow E^{\oplus m}$. The lemma thus reduces to the statement that a general codimension $c$ linear subspace of $\mathbb{P}^{m r-1}$ contains a $\mathbb{P}^{r-1}$ fiber in $S$ if and only if $c r \leqslant m-1$.

Consider the incidence correspondence

$$
J=\left\{(A, H): H \cong \mathbb{P}^{m r-1-c}, A \subset H \cap S \text { is a } \mathbb{P}^{r-1} \text { fiber }\right\} .
$$

The first projection $\pi_{1}$ maps $J$ onto $\mathbb{P}^{m-1}$. The fiber of $\pi_{1}$ over a linear space $A$ is the set of codimension $c$ linear spaces that contain $A$, hence it is isomorphic to the Grassmannian $G((m-1) r-$ 
$c,(m-1) r)$. By the theorem on the dimension of fibers, $J$ is irreducible of dimension $(c r+1)(m-$ $1)-c^{2}$. The second projection cannot dominate $G(m r-c, m r)$ if $\operatorname{dim}(J)<\operatorname{dim}(G(m r-c, m r)=$ $c(m r-c)$. Comparing the two inequalities, we conclude that if $c r>m-1$, the second projection is not dominant. Hence, the general codimension $c$ linear space does not contain a $\mathbb{P}^{r-1}$ fiber in $S$.

To see the converse, we check that if $r \leqslant m-1$, then a general hyperplane contains a codimension $r$ locus of linear $\mathbb{P}^{r-1}$ fibers of $S$. Consider the hyperplane $H$ defined by $\sum_{i=1}^{r} Z_{(i-1) r+i}=0$. Substituting the equations of the Segre embedding, we see that $\sum_{i=1}^{r} \alpha_{i} x_{i}=0$. Since this equation must hold for every choice of $x_{i}$, we conclude $\alpha_{1}=\cdots=\alpha_{r}=0$. Hence, the locus of $\mathbb{P}^{r-1}$ fibers of $S$ contained in $H$ is a codimension $r$ linear space in $\mathbb{P}^{m-1}$. A codimension $c$ linear space is the intersection of $c$ hyperplanes. Moreover, the intersection of $c$ codimension $r$ subvarieties of $\mathbb{P}^{m-1}$ is nonempty if $c r \leqslant m-1$. Hence, if $c r \leqslant m-1$, every codimension $c$ linear space contains a $\mathbb{P}^{r-1}$ fiber of $S$. This suffices to prove the converse.

\subsection{The Picard group and Donaldson-Uhlenbeck-Yau compactification}

Stable vector bundles with $\Delta=\delta(\mu)$ are called height-zero bundles. Their moduli spaces have Picard rank 1 . The ample generator spans the ample cone and there is nothing further to discuss.

For the rest of the subsection, suppose that $\xi=(r, \mu, \Delta)$ is a positive-height Chern character, meaning $\Delta>\delta(\mu)$. There is a pairing on $K\left(\mathbb{P}^{2}\right)$ given by $(\xi, \zeta)=\chi\left(\xi^{*}, \zeta\right)$. When $\Delta>\delta(\mu)$, Drézet proves that the Picard group of $M(\xi)$ is a free abelian group on two generators, naturally identified with $\xi^{\perp}$ in $K\left(\mathbb{P}^{2}\right)$ [LP97]. In $M(\xi)$, linear equivalence and numerical equivalence coincide and the Néron-Severi space $\operatorname{NS}(M(\xi))=\operatorname{Pic}(M(\xi)) \otimes \mathbb{R}$ is a 2-dimensional vector space. In order to specify the ample cone, it suffices to determine its two extremal rays.

In $\xi^{\perp} \cong \operatorname{Pic}(M(\xi))$, there is a unique character $u_{1}$ with $\operatorname{rk}\left(u_{1}\right)=0$ and $c_{1}\left(u_{1}\right)=-r$. The corresponding line bundle $\mathcal{L}_{1}$ is base-point-free and defines the Jun Li morphism $j: M(\xi) \rightarrow$ $M^{D U Y}(\xi)$ to the Donaldson-Uhlenbeck-Yau compactification [HL10, Section 8].

Proposition 2.4. Let $\xi=(r, \mu, \Delta)$ be a positive-height character, and suppose that there are singular sheaves in $M(\xi)$. Then $u_{1}$ spans an extremal edge of $\operatorname{Amp}(M(\xi))$.

Proof. We show that $j$ contracts a curve in $M(\xi)$. Two stable sheaves $E, E^{\prime} \in M(\xi)$ are identified by $j$ if $E^{* *} \cong\left(E^{\prime}\right)^{* *}$ and the sets of singularities of $E$ and $E^{\prime}$ are the same (counting multiplicity). The proof of Theorem 2.2 constructs singular sheaves via an elementary modification that arises from a surjection $E=E_{\alpha}^{\oplus m} \oplus G \rightarrow \mathcal{O}_{p}$. Here $m=0$ if $\Delta-\delta(\mu) \geqslant 1 / r$ or $\mu$ is not exceptional. Otherwise, $1 \leqslant m<r_{\alpha}$. Note that $\operatorname{hom}\left(E, \mathcal{O}_{p}\right)=r$ and $\operatorname{dim}(\operatorname{Aut}(E))=m^{2}+1$ if $G \neq 0$ and $\operatorname{dim}(\operatorname{Aut}(E))=m^{2}$ if $G=0$. Hence, if $r>1$, varying the surjection $E \rightarrow \mathcal{O}_{p}$ gives a positivedimensional family of nonisomorphic Gieseker-semistable sheaves with the same singular support and double dual. If instead $r=1$ and $\Delta \geqslant 2$, then (up to a twist) $j$ is the Hilbert-Chow morphism to the symmetric product, and the result is still true.

Corollary 2.5. Let $\xi=(r, \mu, \Delta)$ be a positive-height character. If $\Delta$ is sufficiently large or if $r \leqslant 6$, then $u_{1}$ spans an edge of $\operatorname{Amp}(M(\xi))$.

Proof. If $\Delta$ is sufficiently large, then this follows immediately from Theorem 2.2 and Proposition 2.4. If $r \leqslant 6$, then without loss of generality we may assume $-\frac{1}{2} \leqslant \mu<\frac{1}{2}$, and the denominator is at most 6 . If $\mu$ is not exceptional, then $\mu \in I_{0}$. Integrality of the Euler characteristic implies that if $\Delta>\delta(\mu)$, then $\Delta \geqslant \delta(\mu)+1 / r$. The corollary then follows from Theorem 2.2 and Proposition 2.4. 


\section{Coskun And J. Huizenga}

\subsection{Bridgeland stability conditions on $\mathbb{P}^{2}$}

We now recall basic facts concerning Bridgeland stability conditions on $\mathbb{P}^{2}$ developed in $[\mathrm{ABCH} 13$, CH14] and [Hui15].

A Bridgeland stability condition $\sigma$ on the bounded derived category $\mathcal{D}^{b}(X)$ of coherent sheaves on a smooth projective variety $X$ is a pair $\sigma=(\mathcal{A}, Z)$, where $\mathcal{A}$ is the heart of a bounded $t$ structure and $Z$ is a group homomorphism

$$
Z: K\left(\mathcal{D}^{b}\left(\mathbb{P}^{2}\right)\right) \rightarrow \mathbb{C}
$$

satisfying the following two properties:

(i) (Positivity) For every object $0 \neq E \in \mathcal{A}$, we have $Z(E) \in\left\{r e^{i \pi \theta} \mid r>0,0<\theta \leqslant 1\right\}$. Positivity allows one to define the slope of a nonzero object in $\mathcal{A}$ by setting

$$
\mu_{Z}(E)=-\frac{\Re(Z(E))}{\Im(Z(E))} .
$$

An object $E$ of $\mathcal{A}$ is called stable (respectively, semistable) if for every proper subobject $F \subset E$ in $\mathcal{A}$ we have $\mu_{Z}(F)<\mu_{Z}(E)$ (respectively, $\mu_{Z}(F) \leqslant \mu_{Z}(E)$ ).

(ii) (Harder-Narasimhan Property) Every object of $\mathcal{A}$ has a finite Harder-Narasimhan filtration.

Bridgeland [Bri08] and Arcara and Bertram [AB13] have constructed Bridgeland stability conditions on projective surfaces. In the case of $\mathbb{P}^{2}$, the relevant Bridgeland stability conditions have the following form. Any torsion-free coherent sheaf $E$ on $\mathbb{P}^{2}$ has a Harder-Narasimhan filtration

$$
0=E_{0} \subset E_{1} \subset \cdots \subset E_{n}=E
$$

with respect to the Mumford slope with semistable factors $\operatorname{gr}_{i}=E_{i} / E_{i-1}$ such that

$$
\mu_{\max }(E)=\mu\left(\operatorname{gr}_{1}\right)>\cdots>\mu\left(\operatorname{gr}_{n}\right)=\mu_{\min }(E) .
$$

Given $s \in \mathbb{R}$, let $\mathcal{Q}_{s}$ be the full subcategory of $\operatorname{coh}\left(\mathbb{P}^{2}\right)$ consisting of sheaves such that the quotient $Q$ by the torsion subsheaf has $\mu_{\min }(Q)>s$. Similarly, let $\mathcal{F}_{s}$ be the full subcategory of $\operatorname{coh}\left(\mathbb{P}^{2}\right)$ consisting of torsion-free sheaves $F$ with $\mu_{\max }(F) \leqslant s$. Then the abelian category

$$
\mathcal{A}_{s}:=\left\{E \in \mathcal{D}^{b}\left(\mathbb{P}^{2}\right): \mathrm{H}^{-1}(E) \in \mathcal{F}_{s}, \mathrm{H}^{0}(E) \in \mathcal{Q}_{s}, H^{i}(E)=0 \text { for } i \neq-1,0\right\}
$$

obtained by tilting the category of coherent sheaves with respect to the torsion pair $\left(\mathcal{F}_{s}, \mathcal{Q}_{s}\right)$ is the heart of a bounded $t$-structure. Let

$$
Z_{s, t}(E)=-\int_{\mathbb{P}^{2}} e^{-(s+i t) H} \operatorname{ch}(E)
$$

where $H$ is the hyperplane class on $\mathbb{P}^{2}$. The pair $\left(\mathcal{A}_{s}, Z_{s, t}\right)$ is a Bridgeland stability condition for every $s>0$ and $t \in \mathbb{R}$. We thus obtain a half-plane of Bridgeland stability conditions on $\mathbb{P}^{2}$ parameterized by $(s, t)$ with $t>0$.

\subsection{Bridgeland walls}

If we fix a Chern character $\xi \in K\left(\mathbb{P}^{2}\right)$, the $(s, t)$-plane of stability conditions for $\mathbb{P}^{2}$ admits a finite wall-and-chamber structure where the objects in $\mathcal{A}_{s}$ with Chern character $\xi$ that are stable with respect to the stability condition $\left(\mathcal{A}_{s}, Z_{s, t}\right)$ remain unchanged within the interior of a chamber [ABCH13, Bri08, BM11, BM14b]. An object $E$ is destabilized by $F$ along a wall $W(E, F)$ if $E$ is semistable on one side of the wall but $F \subset E$ in the category $\mathcal{A}_{s}$ with $\mu_{s, t}(F)>\mu_{s, t}(E)$ on the 
other side of the wall. We call these walls Bridgeland walls. The equations of the wall $W(E, F)$ can be computed using the relation $\mu_{s, t}(F)=\mu_{s, t}(E)$ along the wall.

Suppose that $\xi, \zeta \in K\left(\mathbb{P}^{2}\right) \otimes \mathbb{R}$ are two linearly independent real Chern characters. A potential Bridgeland wall is a set in the $(s, t)$-half-plane of the form

$$
W(\xi, \zeta)=\left\{(s, t): \mu_{s, t}(\xi)=\mu_{s, t}(\zeta)\right\},
$$

where $\mu_{s, t}$ is the slope associated with $\mathcal{Z}_{s, t}$. Bridgeland walls are always potential Bridgeland walls. The potential Bridgeland walls for $\xi$ are all the potential walls $W(\xi, \zeta)$ as $\zeta$ varies in $K\left(\mathbb{P}^{2}\right) \otimes \mathbb{R}$. If $E, F \in D^{b}\left(\mathbb{P}^{2}\right)$, we also write $W(E, F)$ as a shorthand for $W(\operatorname{ch}(E), \operatorname{ch}(F))$.

The potential walls $W(\xi, \zeta)$ can be easily computed in terms of the Chern characters $\xi$ and $\zeta$.

(i) If $\mu(\xi)=\mu(\zeta)$ (where the Mumford slope is interpreted as $\infty$ if the rank is 0 ), then the wall $W(\xi, \zeta)$ is the vertical line $s=\mu(\xi)$ (interpreted as the empty set when the slope is infinite).

(ii) Otherwise, without loss of generality assume that $\mu(\xi)$ is finite, so that $r \neq 0$. The walls $W(\xi, \zeta)$ and $W(\xi, \xi+\zeta)$ are equal, so we may further reduce to the case where both $\xi$ and $\zeta$ have nonzero rank. Then we may encode $\xi=\left(r_{1}, \mu_{1}, \Delta_{1}\right)$ and $\zeta=\left(r_{2}, \mu_{2}, \Delta_{2}\right)$ in terms of slope and discriminant instead of $\mathrm{ch}_{1}$ and $\mathrm{ch}_{2}$. The wall $W(\xi, \zeta)$ is the semicircle centered at the point $(s, 0)$ with

$$
s=\frac{1}{2}\left(\mu_{1}+\mu_{2}\right)-\frac{\Delta_{1}-\Delta_{2}}{\mu_{1}-\mu_{2}}
$$

and having radius $\rho$ given by

$$
\rho^{2}=\left(s-\mu_{1}\right)^{2}-2 \Delta_{1} .
$$

In the principal case of interest, the Chern character $\xi=(r, \mu, \Delta)$ has nonzero rank $r$ and nonnegative discriminant $\Delta$. In this case, the potential walls for $\xi$ consist of a vertical wall $s=\mu$ together with two disjoint nested families of semicircles on either side of this line [ABCH13]. Specifically, for any $s$ with $|s-\mu|>\sqrt{2 \Delta}$, there is a unique semicircular potential wall with center $(s, 0)$ and radius $\rho$ satisfying

$$
\rho^{2}=(s-\mu)^{2}-2 \Delta .
$$

The semicircles are centered along the $s$-axis, with smaller semicircles having centers closer to the vertical wall. Every point in the $(s, t)$-half-plane lies on a unique potential wall for $\xi$. When $r>0$, only the family of semicircles left of the vertical wall is interesting, since an object $E$ with Chern character $\xi$ can only be in categories $\mathcal{A}_{s}$ with $s<\mu$.

Since the number of Bridgeland walls is finite, there exists a largest semicircular Bridgeland wall $W_{\max }$ to the left of the vertical line $s=\mu$ that contains all other semicircular walls. Furthermore, for every $(s, t)$ with $s<\mu$ and contained outside $W_{\max }$, the moduli space of Bridgeland stable objects in $\mathcal{A}_{s}$ with respect to $Z_{s, t}$ and Chern character $\xi$ is isomorphic to the moduli space $M(\xi)[\mathrm{ABCH} 13]$. We call $W_{\max }$ the Gieseker wall.

\subsection{A nef divisor on $M(\xi)$}

Let $(\mathcal{A}, Z)=\sigma_{0} \in W_{\max }$ be a stability condition on the Gieseker wall. Bayer and Macrì [BM14b] construct a nef divisor $\ell_{\sigma_{0}}$ on $M(\xi)$ corresponding to $\sigma_{0}$. They also compute its class and describe geometrically the curves $C \subset M(\xi)$ with $C \cdot \ell_{\sigma_{0}}=0$. 


\section{Coskun And J. Huizenga}

To describe the class of $\ell_{\sigma_{0}}$ in $\xi^{\perp} \cong \operatorname{Pic} M(\xi)$, consider the functional

$$
\begin{aligned}
N^{1}(M(\xi)) & \rightarrow \mathbb{R}, \\
\xi^{\prime} & \mapsto \Im\left(-\frac{Z\left(\xi^{\prime}\right)}{Z(\xi)}\right) .
\end{aligned}
$$

Since the pairing $(\xi, \zeta)=\chi(\xi \otimes \zeta)$ is nondegenerate, we can write this functional as $(\zeta,-)$ for some unique $\zeta \in \xi^{\perp}$. In terms of the isomorphism $\xi^{\perp} \cong \operatorname{Pic} M(\xi)$, we have $\zeta=\left[\ell_{\sigma_{0}}\right]$. Considering $\left(\zeta, \operatorname{ch} \mathcal{O}_{p}\right)$ shows that $\zeta$ has negative rank. Furthermore, if $W_{\max }=W\left(\xi^{\prime}, \xi\right)$ (so that $Z\left(\xi^{\prime}\right)$ and $Z(\xi)$ are real multiples of one another), then $\zeta$ is a negative-rank character in $\left(\xi^{\prime}\right)^{\perp}$. The ray in $N^{1}(M(\xi))$ determined by $\sigma_{0}$ depends only on $W_{\max }$, and not the particular choice of $\sigma_{0}$.

A curve $C \subset M(\xi)$ is orthogonal to $\ell_{\sigma_{0}}$ if and only if two general sheaves parameterized by $C$ are $S$-equivalent with respect to $\sigma_{0}$. This gives an effective criterion for determining when the Bayer-Macrì divisor $\ell_{\sigma_{0}}$ is an extremal nef divisor. In every case where we compute the ample cone of $M(\xi)$, the divisor $\ell_{\sigma_{0}}$ is in fact extremal.

\section{Admissible decompositions}

In this section, we introduce the notion of an admissible decomposition of a Chern character of positive rank. Each such decomposition corresponds to a potential Bridgeland wall. In the cases when we can compute the ample cone, the Gieseker wall will correspond to a certain admissible decomposition.

Definition 3.1. Let $\xi$ be a stable Chern character of positive rank. A decomposition of $\xi$ is a triple $\Xi=\left(\xi^{\prime}, \xi, \xi^{\prime \prime}\right)$ such that $\xi=\xi^{\prime}+\xi^{\prime \prime}$. We say $\Xi$ is an admissible decomposition if furthermore

(D1) the character $\xi^{\prime}$ is semistable;

(D2) the character $\xi^{\prime \prime}$ is stable;

(D3) $0<\operatorname{rk}\left(\xi^{\prime}\right) \leqslant \operatorname{rk}(\xi)$;

(D4) $\mu\left(\xi^{\prime}\right)<\mu(\xi)$;

(D5) if $\operatorname{rk}\left(\xi^{\prime \prime}\right)>0$, then $\mu\left(\xi^{\prime \prime}\right)-\mu\left(\xi^{\prime}\right)<3$.

Remark 3.2. The Chern characters in an admissible decomposition $\Xi$ span a 2-plane in $K\left(\mathbb{P}^{2}\right)$. We write $W(\Xi)$ for the potential Bridgeland wall where characters in this plane have the same slope.

Condition (D1) means that $\xi^{\prime}$ is either semiexceptional or stable. We require $\xi^{\prime \prime}$ to be stable since this holds in all our examples and makes admissibility work better with respect to elementary modifications; see Section 5.

Condition (D5) is useful to control the vanishing of Ext ${ }^{2}$ groups via Serre duality.

There are a couple of numerical properties of decompositions which will frequently arise.

Definition 3.3. Let $\Xi=\left(\xi^{\prime}, \xi, \xi^{\prime \prime}\right)$ be a decomposition.

(i) We say that $\Xi$ is coprime if $\operatorname{rk}(\xi)$ and $c_{1}(\xi)$ are coprime.

(ii) We say that $\Xi$ is torsion if $\operatorname{rk}\left(\xi^{\prime \prime}\right)=0$, and torsion free otherwise. 


\section{ThE AMPLE CONE OF MODUli SPACES OF SHEAVES ON THE PLANE}

The conditions in the definition of an admissible decomposition ensure that there is a wellbehaved space of extensions of the form

$$
0 \rightarrow F \rightarrow E \rightarrow Q \rightarrow 0
$$

with $F \in M\left(\xi^{\prime}\right)$ and $Q \in M\left(\xi^{\prime \prime}\right)$.

Lemma 3.4. Let $\Xi=\left(\xi^{\prime}, \xi, \xi^{\prime \prime}\right)$ be an admissible torsion-free decomposition. We have $\chi\left(\xi^{\prime \prime}, \xi^{\prime}\right)<0$. In particular, for any $F \in M\left(\xi^{\prime}\right)$ and $Q \in M\left(\xi^{\prime \prime}\right)$ there are nonsplit extensions

$$
0 \rightarrow F \rightarrow E \rightarrow Q \rightarrow 0 \text {. }
$$

Furthermore, $\operatorname{Ext}^{1}(Q, F)$ has the expected dimension $-\chi\left(\xi^{\prime \prime}, \xi^{\prime}\right)$ for any $F \in M\left(\xi^{\prime}\right)$ and $Q \in M\left(\xi^{\prime \prime}\right)$.

Proof. From condition (D4) and the torsion-free hypothesis, we have $\mu(\xi)<\mu\left(\xi^{\prime \prime}\right)$. Let $F \in M\left(\xi^{\prime}\right)$ and $Q \in M\left(\xi^{\prime \prime}\right)$. By stability, $\operatorname{Hom}(Q, F)=0$. Using Serre duality with condition (D5), we have $\operatorname{Ext}^{2}(Q, F)=0$. Therefore $\operatorname{ext}^{1}(Q, F)=-\chi\left(\xi^{\prime \prime}, \xi^{\prime}\right)$ and $\chi\left(\xi^{\prime \prime}, \xi^{\prime}\right) \leqslant 0$.

To prove $\chi\left(\xi^{\prime \prime}, \xi^{\prime}\right)<0$, first suppose that $\xi^{\prime}$ is semiexceptional. Then

$$
\chi\left(\xi^{\prime \prime}, \xi^{\prime}\right)=\chi\left(\xi, \xi^{\prime}\right)-\chi\left(\xi^{\prime}, \xi^{\prime}\right)<\chi\left(\xi, \xi^{\prime}\right) .
$$

As in the previous paragraph, $\chi\left(\xi, \xi^{\prime}\right) \leqslant 0$, hence $\chi\left(\xi^{\prime \prime}, \xi^{\prime}\right)<0$. A similar argument works if $\xi^{\prime \prime}$ is semiexceptional.

Assume that neither $\xi^{\prime}$ nor $\xi^{\prime \prime}$ is semiexceptional. Then $-3<\mu\left(\xi^{\prime}\right)-\mu\left(\xi^{\prime \prime}\right)<0$ and $\Delta\left(\xi^{\prime}\right)+$ $\Delta\left(\xi^{\prime \prime}\right)>1$. Since $P(x)<1$ for $-3<x<0$, we conclude $\chi\left(\xi^{\prime \prime}, \xi^{\prime}\right)<0$ by the Riemann-Roch formula.

We now introduce a notion of stability for an admissible decomposition $\Xi$. Let $F_{s^{\prime}} / S^{\prime}$ (respectively, $\left.Q_{s^{\prime \prime}} / S^{\prime \prime}\right)$ be a complete flat family of semistable sheaves with Chern character $\xi^{\prime}$ (respectively, $\xi^{\prime \prime}$ ), parameterized by a smooth and irreducible base variety. Completeness implies that the map $S^{\prime} \rightarrow M\left(\xi^{\prime}\right)$ is dominant (see [LP97, Section 15] for details on complete families). Since $\operatorname{ext}^{1}\left(Q_{s^{\prime \prime}}, F_{s^{\prime}}\right)$ does not depend on $\left(s^{\prime}, s^{\prime \prime}\right) \in S^{\prime} \times S^{\prime \prime}$, there is a projective bundle $S$ over $S^{\prime} \times S^{\prime \prime}$ such that the fiber over a point $\left(s^{\prime}, s^{\prime \prime}\right)$ is $\mathbb{P E x t}^{1}\left(Q_{s^{\prime \prime}}, F_{s^{\prime}}\right)$. Then $S$ is smooth, irreducible, and it carries a universal extension sheaf $E_{s} / S$.

We wish to examine the stability properties of the general extension $E_{s} / S$. If $E_{s}$ is $(\mu-)$ (semi)stable for some $s \in S$, then the general $E_{s}$ has the same stability property. Since the moduli spaces $M\left(\xi^{\prime}\right)$ and $M\left(\xi^{\prime \prime}\right)$ are irreducible, the general $E_{s}$ will be $(\mu-)$ (semi)stable if and only if there exists some extension

$$
0 \rightarrow F \rightarrow E \rightarrow Q \rightarrow 0,
$$

where $F \in M\left(\xi^{\prime}\right), Q \in M\left(\xi^{\prime \prime}\right)$, and $E$ is $(\mu-)$ (semi)stable. By the openness of stability, the existence of some semistable extension is independent of the choice of complete families $S^{\prime}$ and $S^{\prime \prime}$.

Definition 3.5. Let $\Xi$ be an admissible decomposition. We say that $\Xi$ is generically $(\mu-)($ semi)stable if there is some extension

$$
0 \rightarrow F \rightarrow E \rightarrow Q \rightarrow 0
$$

where $F \in M\left(\xi^{\prime}\right), Q \in M\left(\xi^{\prime \prime}\right)$, and $E$ is $(\mu-)($ semi) stable. 


\section{Coskun And J. Huizenga}

\section{Extremal triples}

We now introduce the decomposition of a Chern character $\xi$ which frequently corresponds to the primary edge of the ample cone of $M(\xi)$.

Definition 4.1. We call a triple $\Xi=\left(\xi^{\prime}, \xi, \xi^{\prime \prime}\right)$ of Chern characters extremal if it is an admissible decomposition of $\xi$ with the following additional properties:

(E1) The characters $\xi^{\prime}$ and $\xi$ are slope close: we have $\mu\left(\xi^{\prime}\right)<\mu(\xi)$, and every rational number in the interval $\left(\mu\left(\xi^{\prime}\right), \mu(\xi)\right)$ has denominator greater than $\operatorname{rk}(\xi)$.

(E2) The character $\xi^{\prime}$ is discriminant minimal: if $\theta^{\prime}$ is a stable Chern character with $0<\operatorname{rk}\left(\theta^{\prime}\right) \leqslant$ $\operatorname{rk}(\xi)$ and $\mu\left(\theta^{\prime}\right)=\mu\left(\xi^{\prime}\right)$, then $\Delta\left(\theta^{\prime}\right) \geqslant \Delta\left(\xi^{\prime}\right)$.

(E3) The character $\xi^{\prime}$ is rank minimal: if $\theta^{\prime}$ is a stable Chern character with $\mu\left(\theta^{\prime}\right)=\mu\left(\xi^{\prime}\right)$ and $\Delta\left(\theta^{\prime}\right)=\Delta\left(\xi^{\prime}\right)$, then $\operatorname{rk}\left(\theta^{\prime}\right) \geqslant \operatorname{rk}\left(\xi^{\prime}\right)$.

Remark 4.2. If $\Xi$ is an extremal triple, then it is uniquely determined by $\xi$. The wall $W(\Xi)$ thus also only depends on $\xi$. Not every stable character $\xi$ can be decomposed into an extremal triple $\Xi=\left(\xi^{\prime}, \xi, \xi^{\prime \prime}\right)$, but the vast majority can; see Lemma 4.3 .

Condition (E2) in Definition 3.1 is motivated by the formula for the center $(s, 0)$ of $W(\Xi)$ :

$$
s=\frac{\mu\left(\xi^{\prime}\right)+\mu(\xi)}{2}-\frac{\Delta\left(\xi^{\prime}\right)-\Delta(\xi)}{\mu\left(\xi^{\prime}\right)-\mu(\xi)} .
$$

If $\Delta\left(\xi^{\prime}\right)$ decreases while the other invariants are held fixed, then the center of $W(\Xi)$ moves left. Correspondingly, the wall becomes larger. As we are searching for the largest walls, intuitively we should restrict our attention to triples with minimal $\Delta\left(\xi^{\prime}\right)$.

Similarly, condition (E1) typically helps make the wall $W(\Xi)$ large. In the formula for $s$, the term

$$
-\frac{\Delta\left(\xi^{\prime}\right)-\Delta(\xi)}{\mu\left(\xi^{\prime}\right)-\mu(\xi)}
$$

will dominate the expression if $\Delta(\xi)$ is sufficiently large and $\mu\left(\xi^{\prime}\right)$ is sufficiently close to $\mu(\xi)$.

Condition (E3) forces $\xi^{\prime}$ to be stable, since semiexceptional characters are multiples of exceptional characters.

The next lemma shows that the definition of an extremal triple is not vacuous.

Lemma 4.3. Let $\xi=(r, \mu, \Delta)$ be a stable Chern character, and suppose that

(i) either $\Delta$ is sufficiently large (depending on $r$ and $\mu$ );

(ii) or $r \leqslant 6$.

Then there is a unique extremal triple $\Xi=\left(\xi^{\prime}, \xi, \xi^{\prime \prime}\right)$.

Proof. Let $\left(r^{\bullet}, \mu^{\bullet}, \Delta^{\bullet}\right)$ denote the rank, slope, and discriminant of $\xi^{\bullet}$. The Chern character $\xi^{\prime}$ is uniquely determined by conditions (D1), (D3), and (E1)-(E3); it depends only on $r$ and $\mu$, and not on $\Delta$. Set $\xi^{\prime \prime}=\xi-\xi^{\prime}$, and observe that $r^{\prime \prime}$ and $\mu^{\prime \prime}$ depend only on $r$ and $\mu$. We must check that $\xi^{\prime \prime}$ is stable and $\mu^{\prime \prime}-\mu^{\prime}<3$ if $r^{\prime \prime}>0$. If $r^{\prime \prime}=0$, then $c_{1}\left(\xi^{\prime \prime}\right)>0$, so stability is automatic.

Suppose $r^{\prime \prime}>0$. Let us show $\mu^{\prime \prime}-\mu^{\prime}<3$. By property (E1) we have $\mu^{\prime} \geqslant \mu-1 / r$, so

$$
r^{\prime \prime} \mu^{\prime \prime}=r \mu-r^{\prime} \mu^{\prime} \leqslant\left(r-r^{\prime}\right) \mu+\frac{r^{\prime}}{r}<r^{\prime \prime} \mu+1
$$


and

$$
\mu^{\prime \prime}-\mu^{\prime}<\mu+\frac{1}{r^{\prime \prime}}-\mu+\frac{1}{r}=\frac{1}{r^{\prime \prime}}+\frac{1}{r} \leqslant \frac{3}{2} .
$$

If $r \leqslant 6$, we will see that $\xi^{\prime \prime}$ is stable in Section 6 . Suppose that $\Delta$ is sufficiently large. We have a relation

$$
r \Delta=r^{\prime} \Delta^{\prime}+r^{\prime \prime} \Delta^{\prime \prime}-\frac{r^{\prime} r^{\prime \prime}}{r}\left(\mu^{\prime}-\mu^{\prime \prime}\right)^{2}
$$

The invariants $r^{\prime}, \mu^{\prime}, \Delta^{\prime}, r^{\prime \prime}, \mu^{\prime \prime}$ depend only on $r$ and $\mu$. By making $\Delta$ large, we can make $\Delta^{\prime \prime}$ as large as we want, and thus we can make $\xi^{\prime \prime}$ stable.

Remark 4.4. While the hypotheses of Lemma 4.3 are not as weak as possible, there are stable Chern characters where the lemma fails. Indeed, if $\xi=\left(19, \frac{8}{19}, \frac{184}{361}\right)$, then the unique triple $\left(\xi^{\prime}, \xi, \xi^{\prime \prime}\right)$ satisfying conditions (E1)-(E3) is

$$
\left(\xi^{\prime}, \xi, \xi^{\prime \prime}\right)=\left(\left(12, \frac{5}{12}, \frac{157}{288}\right),\left(19, \frac{8}{19}, \frac{184}{361}\right),\left(7, \frac{3}{7}, \frac{22}{49}\right)\right),
$$

but the triple is not admissible since $\xi^{\prime \prime}$ has $\Delta\left(\xi^{\prime \prime}\right)<\frac{1}{2}$ and so is not stable. In fact, the rank of $\xi$ is minimal in this counterexample.

It is easy to prove a weak stability result for extremal triples.

Proposition 4.5. Let $\Xi=\left(\xi^{\prime}, \xi, \xi^{\prime \prime}\right)$ be an extremal torsion-free triple. Then $\Xi$ is generically $\mu$-semistable.

Proof. By Lemma 3.4, there is a nonsplit extension

$$
0 \rightarrow F \rightarrow E \rightarrow Q \rightarrow 0
$$

with $F \in M^{s}\left(\xi^{\prime}\right)$ stable and $Q \in M(\xi)$. We will show that $E$ is $\mu$-semistable. Since $F$ and $Q$ are torsion free, $E$ is torsion free as well.

Suppose that $E$ is not $\mu$-semistable. Then there is some surjection $E \rightarrow C$ with $\mu(C)<\mu(E)$ and $\operatorname{rk}(C)<\operatorname{rk}(E)$. By passing to a suitable quotient of $C$, we may assume that $C$ is stable. Using slope closeness (E1), we find $\mu(C) \leqslant \mu(F)$.

First, assume $\mu(C)<\mu(F)$. By stability, the composition $F \rightarrow E \rightarrow C$ is 0 , and thus $E \rightarrow C$ induces a map $Q \rightarrow C$. This map is 0 by stability, from which we conclude that $E \rightarrow C$ is 0 , giving a contradiction.

Next, assume $\mu(C)=\mu(F)$. If $\Delta(C)>\Delta(F)$, then we have an inequality $p_{C}<p_{F}$ of reduced Hilbert polynomials, so $F \rightarrow C$ is 0 by stability and we conclude as in the previous paragraph. On the other hand, $\Delta(C)<\Delta(F)$ cannot occur by the minimality condition (E2).

Finally, suppose $\mu(C)=\mu(F)$ and $\Delta(C)=\Delta(F)$. Since $C$ and $F$ are both stable, any nonzero map $F \rightarrow C$ is an isomorphism. Then the composition $E \rightarrow C \rightarrow F$ with the inverse isomorphism splits the sequence.

The following corollary gives the first statement of Theorem 1.4 in the torsion-free case.

Corollary 4.6. If $\Xi$ is a coprime, torsion-free, extremal triple, then it is generically $\mu$-stable.

\section{Elementary modifications}

Many stability properties of an admissible decomposition $\Xi=\left(\xi^{\prime}, \xi, \xi^{\prime \prime}\right)$ are easier to understand when the discriminant $\Delta(\xi)$ is small. Elementary modifications allow us to reduce to the smalldiscriminant case. 


\section{Coskun And J. Huizenga}

Definition 5.1. Let $G$ be a coherent sheaf, and let $G \rightarrow \mathcal{O}_{p}$ be a surjective homomorphism. Then the kernel

$$
0 \rightarrow G^{\prime} \rightarrow G \rightarrow \mathcal{O}_{p} \rightarrow 0
$$

is called an elementary modification of $G$.

If $G$ has positive rank, we observe the equalities

$$
\operatorname{rk}\left(G^{\prime}\right)=\operatorname{rk}(G), \quad \mu\left(G^{\prime}\right)=\mu(G), \quad \Delta\left(G^{\prime}\right)=\Delta(G)+\frac{1}{\operatorname{rk}(G)}, \quad \chi\left(G^{\prime}\right)=\chi(G)-1 .
$$

The next lemma is immediate.

Lemma 5.2. If $G$ is $\mu$-(semi)stable, then any elementary modification of $G$ is $\mu$-(semi)stable.

Warning 5.3. Elementary modifications do not generally preserve Gieseker-(semi)stability. This is our reason for focusing on the $\mu$-stability of extensions.

Given a short exact sequence of sheaves, there is a natural induced sequence involving compatible elementary modifications.

Proposition And Definition 5.4. Suppose that

$$
0 \rightarrow F \rightarrow E \rightarrow Q \rightarrow 0
$$

is a short exact sequence of sheaves. Let $Q^{\prime}$ be the elementary modification of $Q$ corresponding to a homomorphism $Q \rightarrow \mathcal{O}_{p}$, and let $E^{\prime}$ be the elementary modification of $E$ corresponding to the composition $E \rightarrow Q \rightarrow \mathcal{O}_{p}$. Then there is a natural short exact sequence

$$
0 \rightarrow F \rightarrow E^{\prime} \rightarrow Q^{\prime} \rightarrow 0 .
$$

This sequence is called an elementary modification of the original sequence.

Proof. A straightforward argument shows that there is a natural commuting diagram

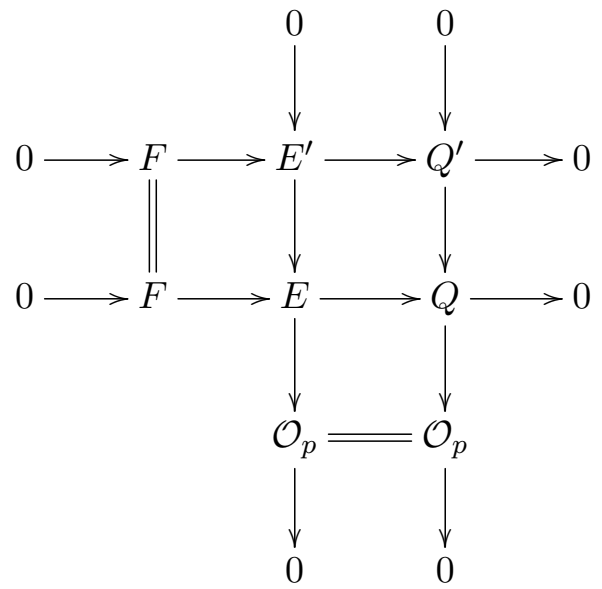

with exact rows and columns.

We similarly extend the notion of elementary modifications to decompositions of Chern characters.

Definition 5.5. Let $\Xi=\left(\xi^{\prime}, \xi, \xi^{\prime \prime}\right)$ be a decomposition. Let $\Theta=\left(\theta^{\prime}, \theta, \theta^{\prime \prime}\right)$ be the decomposition such that 
(i) $\theta^{\prime}=\xi^{\prime}$;

(ii) the characters $\theta$ and $\xi$ have the same rank and slope;

(iii) $\Delta(\theta)=\Delta(\xi)+1 / \operatorname{rk}(\xi)$.

We call $\Theta$ the elementary modification of $\Xi$. If $\Xi$ is admissible, then $\Theta$ is admissible as well.

If $\Xi$ and $\Theta$ are admissible decompositions, we say that $\Theta$ lies above $\Xi$, and write $\Xi \preceq \Theta$, if conditions (i)-(ii) are satisfied and $\Delta(\xi) \leqslant \Delta(\theta)$. Finally, $\Xi$ is minimal if it is a minimal admissible decomposition with respect to $\preceq$.

The next result follows from the integrality of the Euler characteristic and the Riemann-Roch formula.

Lemma 5.6. Let $\Xi$ and $\Theta$ be admissible decompositions. Then $\Xi \preceq \Theta$ if and only if $\Theta$ is an iterated elementary modification of $\Xi$.

Extremality is preserved by elementary modifications.

LEMma 5.7. Suppose that $\Xi$ and $\Theta$ are admissible decompositions with $\Xi \preceq \Theta$. If one decomposition is extremal, then the other is as well.

Combining our results so far in this subsection, we obtain the following tool for proving results on the generic $\mu$-stability of triples.

Proposition 5.8. Suppose that $\Xi$ is a minimal admissible decomposition and that $\Xi$ is generically $\mu$-stable. Then any $\Theta$ which lies above $\Xi$ is also generically $\mu$-stable.

\section{Stability of small-rank extremal triples}

The goal of this subsection is to prove the following theorem.

Theorem 6.1. Let $\Xi=\left(\xi^{\prime}, \xi, \xi^{\prime \prime}\right)$ be an extremal triple with $\operatorname{rk}(\xi) \leqslant 6$. Then $\Xi$ is generically $\mu$-stable.

By Proposition 5.8, we only need to consider cases where $\Xi$ is minimal. We also assume that $\Xi$ is torsion free and defer to Section 7.5 for the torsion case. By twisting, we may assume $0<\mu(\xi) \leqslant 1$. After these reductions, there are a relatively small number of triples to consider. These triples are easily determined by using Lemma 4.3. We list these in order of rank and slope of $\xi$ in Table 1. For each triple, we also indicate the strategy we will use to prove that the triple is generically $\mu$-stable.

Observing that $\xi^{\prime \prime}$ is always stable in Table 1 completes the proof of Lemma 4.3 as promised. The triples labeled "Coprime" are all generically $\mu$-stable by Corollary 4.6. We turn next to the triples labeled "Complete".

Definition 6.2. An admissible decomposition $\Xi$ is called complete if the general $E \in M(\xi)$ can be expressed as an extension

$$
0 \rightarrow F \rightarrow E \rightarrow Q \rightarrow 0
$$

with $F \in M\left(\xi^{\prime}\right)$ and $Q \in M\left(\xi^{\prime \prime}\right)$.

Remark 6.3. Suppose that $\Xi$ is admissible and generically semistable. Recall the universal extension sheaf $E_{s} / S$ discussed before Definition 3.5. If $U \subset S$ is the open subset parameterizing 


\section{Coskun And J. Huizenga}

TABLE 1. The minimal, extremal, torsion-free triples $\Xi=\left(\xi^{\prime}, \xi, \xi^{\prime \prime}\right)=\left(\left(r^{\prime}, \mu^{\prime}, \Delta^{\prime}\right),(r, \mu, \Delta)\right.$, $\left.\left(r^{\prime \prime}, \mu^{\prime \prime}, \Delta^{\prime \prime}\right)\right)$ which must be considered in Theorem 6.1.

\begin{tabular}{cccc}
\hline$\xi^{\prime}$ & $\xi$ & $\xi^{\prime \prime}$ & Strategy \\
\hline$(1,0,0)$ & $\left(2, \frac{1}{2}, \frac{3}{8}\right)$ & $(1,1,1)$ & Coprime \\
$(1,0,0)$ & $\left(3, \frac{1}{3}, \frac{5}{9}\right)$ & $\left(2, \frac{1}{2}, \frac{7}{8}\right)$ & Coprime \\
$\left(2, \frac{1}{2}, \frac{3}{8}\right)$ & $\left(3, \frac{2}{3}, \frac{5}{9}\right)$ & $(1,1,1)$ & Coprime \\
$(1,0,0)$ & $\left(4, \frac{1}{4}, \frac{21}{32}\right)$ & $\left(3, \frac{1}{3}, \frac{8}{9}\right)$ & Coprime \\
$\left(3, \frac{1}{3}, \frac{5}{9}\right)$ & $\left(4, \frac{1}{2}, \frac{5}{8}\right)$ & $(1,1,1)$ & Complete \\
$\left(3, \frac{2}{3}, \frac{5}{9}\right)$ & $\left(4, \frac{3}{4}, \frac{21}{32}\right)$ & $(1,1,1)$ & Coprime \\
$(1,0,0)$ & $\left(5, \frac{1}{5}, \frac{18}{25}\right)$ & $\left(4, \frac{1}{4}, \frac{29}{32}\right)$ & Coprime \\
$\left(3, \frac{1}{3}, \frac{5}{9}\right)$ & $\left(5, \frac{2}{5}, \frac{12}{25}\right)$ & $\left(2, \frac{1}{2}, \frac{3}{8}\right)$ & Coprime \\
$\left(2, \frac{1}{2}, \frac{3}{8}\right)$ & $\left(5, \frac{3}{5}, \frac{12}{25}\right)$ & $\left(3, \frac{2}{3}, \frac{5}{9}\right)$ & Coprime \\
$\left(4, \frac{3}{4}, \frac{21}{32}\right)$ & $\left(5, \frac{4}{5}, \frac{18}{25}\right)$ & $(1,1,1)$ & Coprime \\
$(1,0,0)$ & $\left(6, \frac{1}{6}, \frac{55}{72}\right)$ & $\left(5, \frac{1}{5}, \frac{23}{25}\right)$ & Coprime \\
$\left(4, \frac{1}{4}, \frac{21}{32}\right)$ & $\left(6, \frac{1}{3}, \frac{5}{9}\right)$ & $\left(2, \frac{1}{2}, \frac{3}{8}\right)$ & Complete \\
$\left(5, \frac{2}{5}, \frac{12}{25}\right)$ & $\left(6, \frac{1}{2}, \frac{17}{24}\right)$ & $(1,1,2)$ & Proposition 6.5 \\
$\left(5, \frac{3}{5}, \frac{12}{25}\right)$ & $\left(6, \frac{2}{3}, \frac{5}{9}\right)$ & $(1,1,1)$ & Complete \\
$\left(5, \frac{4}{5}, \frac{18}{25}\right)$ & $\left(6, \frac{5}{6}, \frac{55}{72}\right)$ & $(1,1,1)$ & Coprime \\
\hline
\end{tabular}

semistable sheaves, then $\Xi$ is complete if and only if the moduli map $U \rightarrow M(\xi)$ is dominant. By generic smoothness, $E_{s} / U$ is a complete family of semistable sheaves over a potentially smaller dense open subset.

If $\xi$ is stable, then the general sheaf in $M(\xi)$ is $\mu$-stable by a result of Drézet and Le Potier [DLP85, Corollary 4.12]. Thus if $\Xi$ is complete, then $\Xi$ is generically $\mu$-stable.

Proposition 6.4. Let $\Xi$ be one of the three triples in Table 1 labeled "Complete". Then $\Xi$ is complete, and in particular generically $\mu$-stable.

Proof. First, suppose that $\Xi=\left(\xi^{\prime}, \xi, \xi^{\prime \prime}\right)$ is one of

$$
\left(\left(3, \frac{1}{3}, \frac{5}{9}\right),\left(4, \frac{1}{2}, \frac{5}{8}\right),(1,1,1)\right) \quad \text { or } \quad\left(\left(4, \frac{1}{4}, \frac{21}{32}\right),\left(6, \frac{1}{3}, \frac{5}{9}\right),\left(2, \frac{1}{2}, \frac{3}{8}\right)\right) \text {. }
$$

Let $E$ be a $\mu$-stable sheaf of character $\xi$, and let $Q \in M\left(\xi^{\prime \prime}\right)$ be semistable. We have $\chi(E, Q)>0$ in either case, which implies $\operatorname{hom}(E, Q)>0$ by stability. Pick a nonzero homomorphism $f: E \rightarrow Q$, and let $R \subset Q$ be the image of $f$. By stability considerations, $R$ must have the same rank and slope as $Q$, and $\Delta(R) \geqslant \Delta(Q)$. Letting $F \subset E$ be the kernel of $f$, we find $\operatorname{rk}(F)=\operatorname{rk}\left(\xi^{\prime}\right)$, $\mu(F)=\mu\left(\xi^{\prime}\right)$, and $\Delta(F) \leqslant \Delta\left(\xi^{\prime}\right)$, with equality if and only if $f$ is surjective. Furthermore, $F$ is $\mu$-semistable. Indeed, if there is a subsheaf $G \subset F$ with $\mu(G)>\mu(F)$, then $\mu(G) \geqslant \mu(E)$ by slope closeness (E1), so $G \subset E$ violates the $\mu$-stability of $E$. Then discriminant minimality (E2) forces $\operatorname{ch} F=\xi^{\prime}$. Furthermore, since $\operatorname{rk}\left(\xi^{\prime}\right)$ and $c_{1}\left(\xi^{\prime}\right)$ are coprime, $F$ is actually semistable. Thus $E$ is expressed as an extension

$$
0 \rightarrow F \rightarrow E \rightarrow Q \rightarrow 0
$$

of semistable sheaves, as required. 


\section{The AMPle CONE OF MODUli SPACES OF SHEAVES ON THE Plane}

For the final triple $\left(\left(5, \frac{3}{5}, \frac{12}{25}\right),\left(6, \frac{2}{3}, \frac{5}{9}\right),(1,1,1)\right)$ a slight modification to the previous argument is needed. Fix a $\mu$-stable sheaf $E$ of character $\xi$. This time $\chi\left(\xi, \xi^{\prime \prime}\right)=0$, so the expectation is that if $Q \in M\left(\xi^{\prime \prime}\right)$ is general, then there is no nonzero map $E \rightarrow Q$. Consider the locus

$$
D_{E}=\left\{Q \in M\left(\xi^{\prime \prime}\right): \operatorname{hom}(E, Q) \neq 0\right\} .
$$

Either $D_{E}=M\left(\xi^{\prime \prime}\right)$ or $D_{E}$ is an effective divisor, in which case we can compute its class to show that $D_{E}$ is nonempty. Either way, there is some $Q \in M\left(\xi^{\prime \prime}\right)$ which admits a nonzero homomorphism $E \rightarrow Q$. The argument can now proceed as in the previous cases.

The next proposition treats the last remaining case, completing the proof of Theorem 6.1.

Proposition 6.5. The triple $\Xi=\left(\xi^{\prime}, \xi, \xi^{\prime \prime}\right)=\left(\left(5, \frac{2}{5}, \frac{12}{25}\right),\left(6, \frac{1}{2}, \frac{17}{24}\right),(1,1,2)\right)$ is generically $\mu$-stable

Proof. Observe that $\xi^{\prime}$ is the Chern character of the exceptional bundle $F=E_{2 / 5}$ and $\xi^{\prime \prime}$ is the Chern character of an ideal sheaf $Q=I_{Z}(1)$, where $Z$ has degree 2. Let $Q_{s^{\prime \prime}} / M\left(\xi^{\prime \prime}\right)$ be the universal family. Then the projective bundle $S$ over $M\left(\xi^{\prime \prime}\right)$ with fibers $\mathbb{P} \operatorname{Ext}^{1}\left(Q_{s^{\prime \prime}}, F\right)$ is smooth and irreducible of dimension

$$
\operatorname{dim} S=\operatorname{dim} M\left(\xi^{\prime \prime}\right)-\chi\left(\xi^{\prime \prime}, \xi^{\prime}\right)-1=14,
$$

and there is a universal extension $E_{s} / S$. Since each extension is nonsplit, every $E_{s}$ is $\mu$-semistable by the proof of Proposition 4.5.

A simple computation shows $\operatorname{Hom}\left(F, Q_{s^{\prime \prime}}\right)=0$ for every $Q_{s^{\prime \prime}}$. If $E$ is any sheaf which sits as an extension

$$
0 \rightarrow F \rightarrow E \rightarrow Q_{s^{\prime \prime}} \rightarrow 0
$$

then we apply $\operatorname{Hom}(F,-)$ to conclude $\operatorname{Hom}(F, E) \cong \operatorname{Hom}(F, F)=\mathbb{C}$. Thus the homomorphism $F \rightarrow E$ is unique up to scalars, the sheaf $Q_{s^{\prime \prime}}$ is determined as the cokernel, and since $Q_{s^{\prime \prime}}$ is simple, the corresponding extension class in $\operatorname{Ext}^{1}\left(Q_{s^{\prime \prime}}, F\right)$ is determined up to scalars. We find that distinct points of $S$ parameterize nonisomorphic sheaves. A straightforward computation further shows that the Kodaira-Spencer map $T_{s} S \rightarrow \operatorname{Ext}^{1}\left(E_{s}, E_{s}\right)$ is injective for every $s \in S$.

We now proceed to show that the general $E_{s}$ also satisfies stronger notions of stability.

Step 1: The general $E_{s}$ is semistable. If $E_{s}$ is not semistable, it has a Harder-Narasimhan filtration of length $\ell \geqslant 2$, and all factors have slope $\frac{1}{2}$. For each potential set of numerical invariants of a Harder-Narasimhan filtration, we check that the corresponding Shatz stratum of any $s \in S$ such that the Harder-Narasimhan filtration has that form has positive codimension.

There are only a handful of potential numerical invariants of the filtration. A nonsemistable $E_{s}$ has a semistable subsheaf $G$ with $\mu(G)=\mu(E)=\frac{1}{2}$ and $\Delta(G)<\Delta(E)=\frac{17}{24}$. Then the Chern character of $G$ must be one of

$$
\left(2, \frac{1}{2}, \frac{3}{8}\right), \quad\left(4, \frac{1}{2}, \frac{3}{8}\right), \quad \text { and }\left(4, \frac{1}{2}, \frac{5}{8}\right) \text {. }
$$

We can rule out the first two cases immediately by an ad hoc argument. In either of these cases $E$ has a subsheaf isomorphic to $T_{\mathbb{P}^{2}}(-1)$. Consequently, there is a sequence

$$
0 \rightarrow T_{\mathbb{P}^{2}}(-1) \rightarrow E \rightarrow R \rightarrow 0 .
$$

Applying $\operatorname{Hom}(F,-)$, we see that $\operatorname{Hom}\left(F, T_{\mathbb{P}^{2}}(-1)\right)$ injects into $\operatorname{Hom}(F, E)=\mathbb{C}$. This is absurd, since $\chi\left(F, T_{\mathbb{P}^{2}}(-1)\right)=3$.

Thus the only Shatz stratum we must consider is the locus of sheaves with a filtration

$$
0 \subset G_{1} \subset G_{2}=E_{s}
$$




\section{Coskun And J. Huizenga}

having $\operatorname{chgr}{ }_{1}:=\zeta_{1}=\left(4, \frac{1}{2}, \frac{5}{8}\right)$ and $\operatorname{chgr}_{2}:=\zeta_{2}=\left(2, \frac{1}{2}, \frac{7}{8}\right)$. Let

$$
\Sigma=\operatorname{Flag}\left(E / S ; \zeta_{1}, \zeta_{2}\right) \stackrel{\pi}{\rightarrow} S
$$

be the relative flag variety parameterizing sheaves with a filtration of this form. By the uniqueness of the Harder-Narasimhan filtration, $\pi$ is injective, and its image is the Shatz stratum. The differential of $\pi$ at a point $t=\left(s, G_{1}\right) \in \Sigma$ can be analyzed via the exact sequence

$$
0 \longrightarrow \operatorname{Ext}_{+}^{0}\left(E_{s}, E_{s}\right) \longrightarrow T_{t} \Sigma \stackrel{T_{t} \pi}{\longrightarrow} T_{s} S \stackrel{\omega_{+}}{\longrightarrow} \operatorname{Ext}_{+}^{1}\left(E_{s}, E_{s}\right) .
$$

We have $\operatorname{Ext}_{+}^{0}\left(E_{s}, E_{s}\right)=0$ by [LP97, Proposition 15.3.3], so $T_{t} \pi$ is injective and $\pi$ is an immersion. The codimension of the Shatz stratum near $s$ is at least $r k \omega_{+}$.

The map $\omega_{+}$is the composition $T_{s} S \rightarrow \operatorname{Ext}^{1}\left(E_{s}, E_{s}\right) \rightarrow \operatorname{Ext}_{+}^{1}\left(E_{s}, E_{s}\right)$ of the Kodaira-Spencer map with the canonical map from the long exact sequence of Ext $t_{ \pm}$. The Kodaira-Spencer map is injective, and $\operatorname{Ext}^{1}\left(E_{s}, E_{s}\right) \rightarrow \operatorname{Ext}_{+}^{1}\left(E_{s}, E_{s}\right)$ is surjective since $\operatorname{Ext}_{-}^{2}\left(E_{s}, E_{s}\right)=0$. We have

$$
\operatorname{dim} T_{s} S=14, \quad \operatorname{ext}^{1}\left(E_{s}, E_{s}\right)=16, \quad \text { and } \quad \operatorname{ext}_{+}^{1}\left(E_{s}, E_{s}\right)=-\chi\left(\operatorname{gr}_{1}, \operatorname{gr}_{2}\right)=4,
$$

so we conclude rk $\omega_{+} \geqslant 2$. Therefore the Shatz stratum is a proper subvariety of $S$. We conclude that $\Xi$ is generically semistable.

Step 2: The general $E_{s}$ is $\mu$-stable. Note that a semistable sheaf in $M(\xi)$ is automatically stable. Hence the moduli map $S \rightarrow M^{s}(\xi)$ is injective, and its image has codimension 2 in $M^{s}(\xi)$.

If a sheaf $E \in M^{s}(\xi)$ is not $\mu$-stable, then there is a filtration

$$
0 \subset G_{1} \subset G_{2}=E
$$

such that the quotients $\operatorname{gr}_{i}$ are semistable of slope $\frac{1}{2}$ and $\Delta\left(\operatorname{gr}_{1}\right)>\Delta(E)>\Delta\left(\mathrm{gr}_{2}\right)$ ( see the proof of [DLP85, Theorem 4.11]). Then, as in the previous step, $\zeta_{2}=\operatorname{ch}\left(\mathrm{gr}_{2}\right)$ is one of the characters $(*)$, and $\zeta_{1}=\operatorname{ch}\left(\mathrm{gr}_{1}\right)$ is determined by $\zeta_{2}$. For each of the three possible filtrations, the Shatz stratum in $M^{s}(\xi)$ of sheaves with a filtration of the given form has codimension at least $\operatorname{ext}_{+}^{1}(E, E)=-\chi\left(\mathrm{gr}_{1}, \mathrm{gr}_{2}\right)$.

When $\zeta_{2}=\left(4, \frac{1}{2}, \frac{3}{8}\right)$ we compute $-\chi\left(\operatorname{gr}_{1}, \mathrm{gr}_{2}\right)=6$, and when $\zeta_{2}=\left(4, \frac{1}{2}, \frac{5}{8}\right)$ we have $-\chi\left(\mathrm{gr}_{1}, \mathrm{gr}_{2}\right)=4$. In particular, the corresponding Shatz strata have codimension greater than 2 . On the other hand, for $\zeta_{2}=\left(2, \frac{1}{2}, \frac{3}{8}\right)$ we only find that the stratum has codimension at least 2 , and it is a priori possible that it contains the image of $S \rightarrow M^{s}(\xi)$.

To get around this final problem, we must show that the general sheaf $E_{s}$ parameterized by $S$ does not admit a nonzero map $E_{s} \rightarrow T_{\mathbb{P}^{2}}(-1)$. This can be done by an explicit calculation. Put $Q=I_{Z}(1)$, where $Z=V\left(x, y^{2}\right)$. By stability, $\operatorname{Hom}(Q, T(-1))=0$, so there is an exact sequence

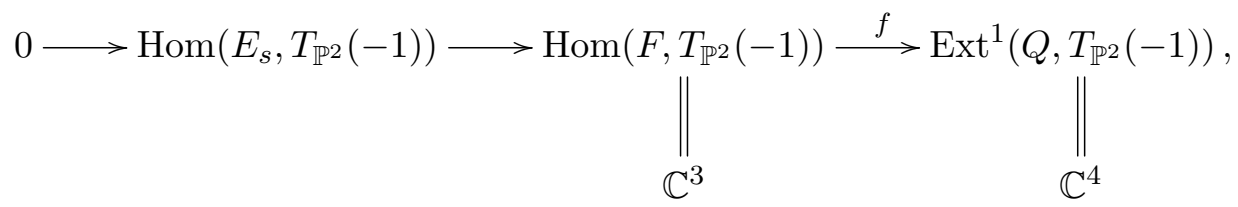

and we must see that $f$ is injective. The map $f$ is the contraction of the canonical map

$$
\operatorname{Ext}^{1}(Q, F) \otimes \operatorname{Hom}\left(F, T_{\mathbb{P}^{2}}(-1)\right) \rightarrow \operatorname{Ext}^{1}\left(Q, T_{\mathbb{P}^{2}}(-1)\right)
$$

corresponding to the extension class of $E$ in $\operatorname{Ext}^{1}(Q, F)$. This canonical map can be explicitly 
The Ample Cone of MOduli Spaces of SheAves on the Plane

computed using the standard resolutions

$$
\begin{aligned}
& 0 \longrightarrow \mathcal{O}_{\mathbb{P}^{2}}(-2) \longrightarrow \mathcal{O}_{\mathbb{P}^{2}}^{6} \longrightarrow \longrightarrow \\
& 0 \longrightarrow \mathcal{O}_{\mathbb{P}^{2}}(-2) \longrightarrow \mathcal{O}_{\mathbb{P}^{2}}(-1) \oplus \mathcal{O}_{\mathbb{P}^{2}} \longrightarrow 0 \\
& 0 \longrightarrow \mathcal{O}_{\mathbb{P}^{2}}(-1) \longrightarrow T_{\mathbb{P}^{2}}(-1) \longrightarrow 0,
\end{aligned}
$$

with the special form of $Q$ simplifying the calculation. The injectivity of $f$ for a general $E_{s}$ follows easily from this computation.

\section{Curves of extensions}

\subsection{General results}

Let $F$ and $Q$ be sheaves, and suppose that the general extension $E$ of $Q$ by $F$ is semistable of Chern character $\xi$. In this section, we study the moduli map

$$
\mathbb{P} \operatorname{Ext}^{1}(Q, F) \rightarrow M(\xi) .
$$

In particular, we would like to be able to show that this map is nonconstant.

DeFinition 7.1. Let $\Xi$ be a generically semistable admissible decomposition. We say that $\Xi$ gives curves if for general $F \in M\left(\xi^{\prime}\right)$ and $Q \in M\left(\xi^{\prime \prime}\right)$, the map $\mathbb{P} \operatorname{Ext}^{1}(Q, F)-\rightarrow M(\xi)$ is nonconstant.

There are three essential ways that $\Xi$ could fail to give curves:

(i) If $-\chi\left(\xi^{\prime \prime}, \xi^{\prime}\right)=1$, then $\mathbb{P} \operatorname{Ext}^{1}(Q, F)$ is a point.

(ii) The sheaves parameterized by $\mathbb{P} \operatorname{Ext}^{1}(Q, F)$ might all be strictly semistable and $S$-equivalent.

(iii) The sheaves parameterized by $\mathbb{P} \operatorname{Ext}^{1}(Q, F)$ might all be isomorphic.

Possibility (i) is easy to check for any given triple. If $\Xi$ is generically stable, then possibility (ii) cannot arise when $F$ and $Q$ are general, so this is also easy to rule out. The third case requires the most work to deal with.

Lemma 7.2. Let $F$ and $Q$ be simple sheaves with $\operatorname{Hom}(F, Q)=0$. Then distinct points of $\mathbb{P} \operatorname{Ext}^{1}(Q, F)$ parameterize nonisomorphic sheaves.

Proof. Suppose that $E$ is a sheaf which can be realized as an extension

$$
0 \rightarrow F \rightarrow E \rightarrow Q \rightarrow 0 \text {. }
$$

Since $F$ is simple and $\operatorname{Hom}(F, Q)=0$, we find $\operatorname{hom}(F, E)=1$. Similarly, since $Q$ is simple and $\operatorname{Hom}(F, Q)=0$, we have $\operatorname{hom}(E, Q)=1$. This means that the corresponding class in $\mathbb{P} \operatorname{Ext}^{1}(Q, F)$ depends only on the isomorphism class of $E$.

The lemma gives us a simple criterion for proving that a triple $\Xi$ gives curves.

Proposition 7.3. Let $\Xi=\left(\xi^{\prime}, \xi, \xi^{\prime \prime}\right)$ be an admissible, generically stable triple, and assume that $\xi^{\prime}$ is stable. Suppose that

(i) either $\Xi$ is not minimal;

(ii) or $-\chi\left(\xi^{\prime \prime}, \xi^{\prime}\right) \geqslant 2$.

If $\operatorname{Hom}(F, Q)=0$ for a general $F \in M\left(\xi^{\prime}\right)$ and $Q \in M\left(\xi^{\prime \prime}\right)$, then $\Xi$ gives curves. 


\section{Coskun And J. Huizenga}

Proof. If $\Xi$ is not minimal, then $-\chi\left(\xi^{\prime \prime}, \xi^{\prime}\right) \geqslant 2$ holds automatically. Indeed, since $\Xi$ is not minimal, it is an elementary modification of another admissible triple $\Theta=\left(\theta^{\prime}, \theta, \theta^{\prime \prime}\right)$. Then $\chi\left(\xi^{\prime \prime}, \xi^{\prime}\right)<\chi\left(\theta^{\prime \prime}, \theta^{\prime}\right)<0$ by the Riemann-Roch formula and Lemma 3.4.

Since $\xi^{\prime}$ and $\xi^{\prime \prime}$ are stable, we can choose stable sheaves $F \in M\left(\xi^{\prime}\right)$ and $Q \in M\left(\xi^{\prime \prime}\right)$ such that $\operatorname{Hom}(F, Q)=0$, and the general extension of $Q$ by $F$ is stable. By Lemma 7.2, the triple $\Xi$ gives curves.

We also observe that elementary modifications behave well with respect to the notion of giving curves.

Lemma 7.4. Suppose that $\Xi=\left(\xi^{\prime}, \xi, \xi^{\prime \prime}\right)$ is admissible and generically $\mu$-stable. If $\Xi \preceq \Theta$ and $\Xi$ gives curves, then $\Theta$ gives curves.

Proof. Suppose that $\Theta$ is obtained from $\Xi$ by a single elementary modification. Let $F \in M\left(\xi^{\prime}\right)$ and $Q \in M\left(\xi^{\prime \prime}\right)$ be general. Take $U \subset \mathbb{P} \operatorname{Ext}^{1}(Q, F)$ to be the dense open subset parameterizing $\mu$-stable sheaves $E$ which are locally free at a general fixed point $p \in \operatorname{Supp} Q$. Let $Q \rightarrow \mathcal{O}_{p}$ be a surjective homomorphism. Given any extension

$$
0 \rightarrow F \rightarrow E \rightarrow Q \rightarrow 0
$$

corresponding to a point of $U$, we get an exact sequence of compatible elementary modifications

$$
0 \rightarrow F \rightarrow E^{\prime} \rightarrow Q^{\prime} \rightarrow 0
$$

as in Definition 5.4. As $E$ can be recovered from $E^{\prime}$ and the map $U \rightarrow M(\xi)$ is nonconstant, we conclude that the map $\mathbb{P} \operatorname{Ext}^{1}\left(Q^{\prime}, F\right) \rightarrow M(\theta)$ is nonconstant. Thus $\Theta$ gives curves.

\subsection{Curves from coprime triples with large discriminant}

Our next result provides the dual curves we will need to prove Theorem 1.1.

Theorem 7.5. Let $\Xi=\left(\xi^{\prime}, \xi, \xi^{\prime \prime}\right)$ be a coprime extremal triple, and suppose that $\Delta(\xi)$ is sufficiently large, depending on $\operatorname{rk}(\xi)$ and $\mu(\xi)$. Then $\Xi$ gives curves.

Proof. The triple $\Xi$ is not minimal, since $\Delta(\xi)$ is large. By Proposition 7.3 , we only need to show that if $F \in M\left(\xi^{\prime}\right)$ and $Q \in M\left(\xi^{\prime \prime}\right)$ are general and $\Delta(\xi)$ is sufficiently large, then $\operatorname{Hom}(F, Q)=0$. Fix general $F \in M\left(\xi^{\prime}\right)$ and $Q \in M\left(\xi^{\prime \prime}\right)$. If $\operatorname{Hom}(F, Q) \neq 0$, choose a nonzero homomorphism $F \rightarrow Q$. We can find a surjective homomorphism $Q \rightarrow \mathcal{O}_{p}$ such that $F \rightarrow Q \rightarrow \mathcal{O}_{p}$ is also surjective. Then applying $\operatorname{Hom}(F,-)$ to the elementary modification sequence

$$
0 \rightarrow Q^{\prime} \rightarrow Q \rightarrow \mathcal{O}_{p} \rightarrow 0
$$

we find that $\operatorname{Hom}\left(F, Q^{\prime}\right)$ is a proper subspace of $\operatorname{Hom}(F, Q)$. Repeating this process, we can find some $\Theta \succeq \Xi$ such that $\operatorname{Hom}(F, Q)=0$ for general $F \in M\left(\theta^{\prime}\right)$ and $Q \in M\left(\theta^{\prime \prime}\right)$. Then $\Theta$ gives curves, and by Lemma 7.4 any $\Lambda \succeq \Theta$ also gives curves.

\subsection{Curves from small-rank triples}

We now discuss extremal curves in the moduli space $M(\xi)$ when the rank is small. For all but a handful of characters $\xi$ we can apply the next theorem.

Theorem 7.6. Let $\Xi=\left(\xi^{\prime}, \xi, \xi^{\prime \prime}\right)$ be an extremal triple with $\operatorname{rk}(\xi) \leqslant 6$. Suppose $\chi\left(\xi^{\prime}, \xi^{\prime \prime}\right) \leqslant 0$. Then $\Xi$ gives curves. 
The Ample cone of moduli spaces of sheaves on the Plane

TABLE 2. Triples to be considered for the proof of Theorem 7.6

\begin{tabular}{cccccccc}
\hline$\xi^{\prime}$ & $\xi$ & $\xi^{\prime \prime}$ & $\chi\left(\xi^{\prime}, \xi^{\prime \prime}\right)$ & $\xi^{\prime}$ & $\xi$ & $\xi^{\prime \prime}$ & $\chi\left(\xi^{\prime}, \xi^{\prime \prime}\right)$ \\
\hline$(1,0,0)$ & $\left(2, \frac{1}{2}, \frac{11}{8}\right)$ & $(1,1,3)$ & 0 & $\left(2, \frac{1}{2}, \frac{3}{8}\right)$ & $\left(5, \frac{3}{5}, \frac{17}{25}\right)$ & $\left(3, \frac{2}{3}, \frac{8}{9}\right)$ & 0 \\
$(1,0,0)$ & $\left(3, \frac{1}{3}, \frac{11}{9}\right)$ & $\left(2, \frac{1}{2}, \frac{15}{8}\right)$ & 0 & $\left(4, \frac{3}{4}, \frac{21}{32}\right)$ & $\left(5, \frac{4}{5}, \frac{18}{25}\right)$ & $(1,1,1)$ & -1 \\
$\left(2, \frac{1}{2}, \frac{3}{8}\right)$ & $\left(3, \frac{2}{3}, \frac{8}{9}\right)$ & $(1,1,2)$ & -1 & $(1,0,0)$ & $\left(6, \frac{1}{6}, \frac{79}{72}\right)$ & $\left(5, \frac{1}{5}, \frac{33}{25}\right)$ & 0 \\
$(1,0,0)$ & $\left(4, \frac{1}{4}, \frac{37}{32}\right)$ & $\left(3, \frac{1}{3}, \frac{14}{9}\right)$ & 0 & $\left(4, \frac{1}{4}, \frac{21}{32}\right)$ & $\left(6, \frac{1}{3}, \frac{13}{18}\right)$ & $\left(2, \frac{1}{2}, \frac{7}{8}\right)$ & -1 \\
$\left(3, \frac{1}{3}, \frac{5}{9}\right)$ & $\left(4, \frac{1}{2}, \frac{7}{8}\right)$ & $(1,1,2)$ & -1 & $\left(5, \frac{2}{5}, \frac{12}{25}\right)$ & $\left(6, \frac{1}{2}, \frac{17}{24}\right)$ & $(1,1,2)$ & -2 \\
$\left(3, \frac{2}{3}, \frac{5}{9}\right)$ & $\left(4, \frac{3}{4}, \frac{21}{32}\right)$ & $(1,1,1)$ & 0 & $\left(5, \frac{3}{5}, \frac{12}{25}\right)$ & $\left(6, \frac{2}{3}, \frac{13}{18}\right)$ & $(1,1,2)$ & -4 \\
$(1,0,0)$ & $\left(5, \frac{1}{5}, \frac{28}{25}\right)$ & $\left(4, \frac{1}{4}, \frac{45}{32}\right)$ & 0 & $\left(5, \frac{4}{5}, \frac{18}{25}\right)$ & $\left(6, \frac{5}{6}, \frac{55}{72}\right)$ & $(1,1,1)$ & -2 \\
$\left(3, \frac{1}{3}, \frac{5}{9}\right)$ & $\left(5, \frac{2}{5}, \frac{17}{25}\right)$ & $\left(2, \frac{1}{2}, \frac{7}{8}\right)$ & -1 & & & & \\
\hline
\end{tabular}

Proof. As with the proof of Theorem 6.1, we assume $0<\mu(\xi) \leqslant 1$. By Lemma 7.4, it is enough to consider triples $\Xi$ such that any admissible $\Theta$ with $\Theta \prec \Xi$ has $\chi\left(\theta^{\prime}, \theta^{\prime \prime}\right)>0$. We also assume that $\Xi$ is torsion free, and handle the torsion case in Section 7.5. We list the relevant triples together with $\chi\left(\xi^{\prime}, \xi^{\prime \prime}\right)$ in Table 2 .

Every triple in the table satisfies $\chi\left(\xi^{\prime \prime}, \xi^{\prime}\right) \leqslant-2$. To apply Proposition 7.3 , we need to see that if $F \in M\left(\xi^{\prime}\right)$ and $Q \in M\left(\xi^{\prime \prime}\right)$ are general, then $\operatorname{Hom}(F, Q)=0$. This can easily be checked case by case via standard sequences or by Macaulay2. We omit the details.

\subsection{Sporadic small-rank triples}

Here we discuss the few sporadic Chern characters not addressed by Theorem 7.6. Suppose that $\xi$ has positive height and $\operatorname{rk}(\xi) \leqslant 6$, and let $\Xi=\left(\xi^{\prime}, \xi, \xi^{\prime \prime}\right)$ be extremal. If $\Xi$ is torsion free and $\chi\left(\xi^{\prime}, \xi^{\prime \prime}\right)>0$, then $\xi^{\prime}=(1,0,0)$ and

$$
\xi \in\left\{\left(2, \frac{1}{2}, \frac{7}{8}\right),\left(3, \frac{1}{3}, \frac{8}{9}\right),\left(4, \frac{1}{4}, \frac{29}{32}\right),\left(5, \frac{1}{5}, \frac{23}{25}\right),\left(6, \frac{1}{6}, \frac{67}{72}\right)\right\} .
$$

That is,

$$
\xi=\left(r, \frac{1}{r}, P\left(-\frac{1}{r}\right)+\frac{1}{r}\right)
$$

for some $r$ with $2 \leqslant r \leqslant 6$. In this case, we have $\chi\left(\xi^{\prime}, \xi\right)=2$, so the general sheaf $E \in M(\xi)$ admits two maps from $\mathcal{O}_{\mathbb{P}^{2}}$. Along the wall $W(\Xi)$, the destabilizing subobject of $E$ should therefore be $\mathcal{O}_{\mathbb{P}^{2}}^{2}$ instead of $\mathcal{O}_{\mathbb{P}^{2}}$. Furthermore, assuming that there are sheaves $E \in M(\xi)$ which are Bridgeland stable just outside $W(\Xi)$, the wall $W(\Xi)$ must be the collapsing wall. We will show in the next section that $W(\Xi)$ is actually the Gieseker wall. This implies that the primary edges of the ample and effective cones of divisors on $M(\xi)$ coincide. Our study of the effective cone in [CHW15] easily implies the next result.

Proposition 7.7. Let $r \geqslant 2$, and let $\Xi=\left(\xi^{\prime}, \xi, \xi^{\prime \prime}\right)$ be the admissible decomposition with $\xi^{\prime}=(2,0,0)$ and $\xi=(r, 1 / r, P(-1 / r)+(1 / r))$. Then $\Xi$ is complete and gives curves.

While we had to modify the original extremal triple $\Xi$ in order to make it give curves, note that the wall $W(\Xi)$ is unchanged by this modification. When showing that $W(\Xi)$ is the largest wall in Section 8, we will not need to handle these cases separately.

In the case of the Chern character $\xi=\left(6, \frac{1}{3}, \frac{13}{18}\right)$, Theorem 7.6 shows that the corresponding extremal triple $\Xi$ gives curves. However, the wall $W(\Xi)$ is actually empty. In this case $\chi\left(\mathcal{O}_{\mathbb{P}^{2}}, E\right)=5$ for $E \in M(\xi)$ and the Chern character $\xi^{\prime}=(5,0,0)$ will correspond to the primary 


\section{Coskun And J. Huizenga}

edges of both the effective and ample cones. Again, curves dual to this edge of the effective cone are given by [CHW15].

Proposition 7.8. The admissible decomposition $\Xi=\left((5,0,0),\left(6, \frac{1}{3}, \frac{13}{18}\right),(1,2,6)\right)$ is complete and gives curves.

\subsection{Torsion triples}

Let $\Xi=\left(\xi^{\prime}, \xi, \xi^{\prime \prime}\right)$ be an extremal torsion triple, and let $r=\operatorname{rk}(\xi)=\operatorname{rk}\left(\xi^{\prime}\right)$. We have $\mu(\xi)-\mu\left(\xi^{\prime}\right)=$ $1 / r$ by the slope-closeness condition (E1). Write

$$
\mu\left(\xi^{\prime}\right)=\frac{a}{b} \quad \text { and } \quad \mu(\xi)=\frac{c}{d}
$$

in lowest terms. The numbers $\mu\left(\xi^{\prime}\right)$ and $\mu(\xi)$ are consecutive terms in the Farey sequence of order $r$, so $\mu(\xi)-\mu\left(\xi^{\prime}\right)=1 / b d$ and we deduce that $b d=r$. Also, the mediant

$$
\frac{a+c}{b+d}
$$

must have denominator $b+d$ greater than $r$. The two conditions $b d=r$ and $b+d>r$ together imply that either $b=1$ or $d=1$. That is, either $\mu\left(\xi^{\prime}\right)$ or $\mu(\xi)$ is an integer.

If $\mu\left(\xi^{\prime}\right)$ is an integer, then by discriminant minimality (E2) and rank minimality (E3) we have $\xi^{\prime}=\left(1, \mu\left(\xi^{\prime}\right), 0\right)$. Thus $r=1$, and in every case $\mu(\xi)$ is also an integer.

We may assume $\mu(\xi)=1$. Consider the triple $\Xi$ with

$$
\xi^{\prime}=\left(r, 1-\frac{1}{r}, P\left(-\frac{1}{r}\right)\right) \quad \text { and } \quad \xi=(r, 1,1) .
$$

The character $\xi^{\prime \prime}$ is then $\operatorname{ch} \mathcal{O}_{L}$, where $L \subset \mathbb{P}^{2}$ is a line. We have $\chi\left(\xi, \xi^{\prime \prime}\right)=0$, and $\Xi$ is complete (hence generically $\mu$-stable) by a similar argument to Proposition 6.4. If $r \geqslant 2$, then

$$
\operatorname{dim} M\left(\xi^{\prime}\right)+\operatorname{dim} M\left(\xi^{\prime \prime}\right)=\left(r^{2}-3 r+2\right)+2<r^{2}+1=\operatorname{dim} M(\xi),
$$

so $\Xi$ must give curves. When $r=1$, for any $p \in L$ there is a sequence

$$
0 \rightarrow \mathcal{O}_{\mathbb{P}^{2}} \rightarrow I_{p}(1) \rightarrow \mathcal{O}_{L} \rightarrow 0
$$

so $\Xi$ gives curves when $r=1$ as well. Applying elementary modifications, we conclude our discussion with the following.

Proposition 7.9. Let $\Xi=\left(\xi^{\prime}, \xi, \xi^{\prime \prime}\right)$ be a torsion extremal triple. If $\xi$ is not the Chern character of a line bundle, then $\Xi$ is generically $\mu$-stable and gives curves.

\section{The ample cone}

\subsection{Notation}

We begin by fixing notation for the rest of the paper. Let $\xi$ be a stable Chern character of positive height. We assume that one of the following three hypotheses holds:

(H1) The integers $\operatorname{rk}(\xi)$ and $c_{1}(\xi)$ are coprime and $\Delta(\xi)$ is sufficiently large.

(H2) We have $\operatorname{rk}(\xi) \leqslant 6$, there are no strictly semistable sheaves of character $\xi$, and $\xi$ is not a twist of $\left(6, \frac{1}{3}, \frac{13}{18}\right)$.

(H3) $\xi=\left(6, \frac{1}{3}, \frac{13}{18}\right)$.

Suppose that we are in case (H1) or (H2). There is an extremal triple $\Xi=\left(\xi^{\prime}, \xi, \xi^{\prime \prime}\right)$. Either $\Xi$ gives curves or we are in one of the cases of Proposition 7.7, in which case there is a decomposition 


\section{ThE AMPLE CONE OF MODUli SPACES OF SHEAVES ON THE PLANE}

of $\xi$ which gives curves and has the same corresponding wall. As discussed in Section 2.6, to show that the primary edge of the ample cone corresponds to $W(\Xi)$ it will be enough to show that $W(\Xi)$ is the Gieseker wall $W_{\max }$. Note that $W_{\max }$ cannot be strictly nested inside $W(\Xi)$, since then by our work so far there are sheaves $E \in M(\xi)$ destabilized along $W(\Xi)$. We must show that $W_{\max }$ is not larger than $W(\Xi)$.

Let $E \in M(\xi)$ be a sheaf which is destabilized along some wall. This determines an exact sequence

$$
0 \rightarrow F \rightarrow E \rightarrow Q \rightarrow 0
$$

such that for any $(s, t)$ on the wall the objects $E, F$, and $Q$ are $\sigma_{s, t}$-semistable of the same slope [ABCH13, Section 6]. Furthermore, the sequence is exact in any of the corresponding categories $\mathcal{A}_{s}$ along the wall. Above the wall, $\mu_{s, t}(F)<\mu_{s, t}(E)$, and below the wall the inequality is reversed. Let $\Theta=\left(\theta^{\prime}, \theta, \theta^{\prime \prime}\right)=(\operatorname{ch} F, \operatorname{ch} E, \operatorname{ch} Q)$ be the corresponding decomposition of $\xi=\theta$, so that the wall is $W(\Theta)$. Our job is to show that $W(\Theta)$ is no larger than $W(\Xi)$ by imposing numerical restrictions on $\theta^{\prime}$. We begin by imposing some easy restrictions on $\theta^{\prime}$.

Lemma 8.1. The object $F$ is a nonzero torsion-free sheaf, so $\operatorname{rk}(F) \geqslant 1$. We have $\mu(F)<\mu(E)$, and every Harder-Narasimhan factor of $F$ has slope at most $\mu(E)$.

Proof. Fix a category $\mathcal{A}_{s}$ along $W(\Theta)$. Taking cohomology sheaves of the destabilizing sequence of $E$, we get a long exact sequence

$$
0 \rightarrow \mathrm{H}^{-1}(F) \rightarrow 0 \rightarrow \mathrm{H}^{-1}(Q) \rightarrow \mathrm{H}^{0}(F) \rightarrow \mathrm{H}^{0}(E) \rightarrow \mathrm{H}^{0}(Q) \rightarrow 0
$$

because $E \in \mathcal{Q}_{s}$. Thus $F$ is a sheaf in $\mathcal{Q}_{s}$. We write $K=\mathrm{H}^{-1}(Q)$ and $C=\mathrm{H}^{0}(Q)$, so $K \in \mathcal{F}_{s}$, $C \in \mathcal{Q}_{s}$, and we have an exact sequence of sheaves

$$
0 \rightarrow K \rightarrow F \rightarrow E \rightarrow C \rightarrow 0 .
$$

Since $E$ is torsion free, the torsion subsheaf of $F$ is contained in $K$. Since $K \in \mathcal{F}_{s}$ is torsion free, we conclude that $F$ is torsion free. Clearly also $F$ is nonzero, for otherwise $\left(\theta^{\prime}, \theta, \theta^{\prime \prime}\right)$ would not span a 2-plane in $K\left(\mathbb{P}^{2}\right)$. We conclude $\operatorname{rk}(F) \geqslant 1$.

Let

$$
\{0\} \subset F_{1} \subset \cdots \subset F_{\ell}=F
$$

be the Harder-Narasimhan filtration of $F$. If $\mu\left(F_{1}\right)>\mu(E)$, then $F_{1} \rightarrow E$ is 0 and $F_{1} \subset K$. Since $K \in \mathcal{F}_{s}$ for any $s$ along $W(\Theta)$, this is absurd. Therefore $\mu\left(F_{1}\right) \leqslant \mu(E)$. We cannot have $\mu(F)=\mu(E)$ since then $W(\Theta)$ would be the vertical wall, so we conclude $\mu(F)<\mu(E)$.

\subsection{Excluding higher-rank walls}

In this subsection, we bound the rank of $F$ under the assumption that $W(\Theta)$ is larger than $W(\Xi)$. In general, there will be walls corresponding to "higher rank" subobjects. We show that the Gieseker wall cannot correspond to such a subobject.

TheOrem 8.2. Keep the notation and hypotheses from above.

(i) Suppose that hypothesis $(H 1)$ or $(H 2)$ holds. If $W(\Theta)$ is larger than $W(\Xi)$, then $1 \leqslant \operatorname{rk}\left(\theta^{\prime}\right) \leqslant$ $\operatorname{rk}(\xi)$.

(ii) If $\xi=\left(6, \frac{1}{3}, \frac{13}{18}\right)$, then the same result holds for the decomposition $\Xi=\left(\xi^{\prime}, \xi, \xi^{\prime \prime}\right)$ with $\xi^{\prime}=(5,0,0)$.

The next inequality is our main tool for proving the theorem. 


\section{Coskun And J. Huizenga}

Proposition 8.3. If $\operatorname{rk}(F)>\operatorname{rk}(E)$, then the radius $\rho_{\Theta}$ of $W(\Theta)$ satisfies

$$
\rho_{\Theta}^{2} \leqslant \frac{\operatorname{rk}(E)^{2}}{2(\operatorname{rk}(E)+1)} \Delta(E) .
$$

Proof. Consider the exact sequence of sheaves

$$
0 \rightarrow K^{k} \rightarrow F^{f} \rightarrow E^{e} \rightarrow C^{c} \rightarrow 0
$$

with the superscripts denoting the ranks of the sheaves. Since $F$ is in the categories $\mathcal{Q}_{s}$ along $W(\Theta)$, we have

$$
f\left(s_{\Theta}+\rho_{\Theta}\right) \leqslant f \mu(F)=c_{1}(F)=c_{1}(K)+c_{1}(E)-c_{1}(C)=k \mu(K)+e \mu(E)-c_{1}(C) .
$$

Next, since $K$ is nonzero and in $\mathcal{F}_{s}$ along $W(\Theta)$, we have $\mu(K) \leqslant s_{\Theta}-\rho_{\Theta}$, and thus

$$
f\left(s_{\Theta}+\rho_{\Theta}\right) \leqslant k\left(s_{\Theta}-\rho_{\Theta}\right)+e \mu(E)-c_{1}(C) .
$$

Rearranging gives

$$
(k+f) \rho_{\Theta} \leqslant(k-f) s_{\Theta}+e \mu(E)-c_{1}(C) .
$$

If $C$ is zero or torsion, then $k-f=-e$ and $c_{1}(C) \geqslant 0$, from which we get

$$
(k+f) \rho_{\Theta} \leqslant(k-f)\left(s_{\Theta}-\mu(E)\right) .
$$

This inequality also holds if $C$ is not torsion. In that case, we have $k-f=c-e$ and since $E$ is semistable, $c_{1}(C)=c \mu(C) \geqslant c \mu(E)$, from which the inequality follows.

Both sides of inequality (8.1) are positive, and squaring both sides gives

$$
(k+f)^{2} \rho_{\Theta}^{2} \leqslant(k-f)^{2}\left(\rho_{\Theta}^{2}+2 \Delta(E)\right) .
$$

We conclude

$$
\rho_{\Theta}^{2} \leqslant \frac{(k-f)^{2}}{2 k f} \Delta(E)
$$

This inequality is as weak as possible when the coefficient $(k-f)^{2} /(2 k f)$ is maximized. Viewing $e$ as fixed, $k$ and $f$ are integers satisfying $f \geqslant e+1$ and $f-e \leqslant k \leqslant f$. It is easy to see that the coefficient is maximized when $f=e+1$ and $k=1$, which corresponds to the inequality we wanted to prove.

Proof of Theorem 8.2. We recall that

$$
\rho_{\Xi}^{2}=\left(\frac{\mu\left(\xi^{\prime}\right)-\mu(\xi)}{2}-\frac{\Delta(\xi)-\Delta\left(\xi^{\prime}\right)}{\mu(\xi)-\mu\left(\xi^{\prime}\right)}\right)^{2}-2 \Delta(\xi) .
$$

If we view $\rho_{\Xi}^{2}$ as a function of $\Delta(\xi)$, then it grows quadratically as $\Delta(\xi)$ increases. Suppose that $\Delta(\xi)$ is large enough so that

$$
\rho_{\Xi}^{2} \geqslant \frac{\operatorname{rk}(\xi)^{2}}{2(\operatorname{rk}(\xi)+1)} \Delta(\xi) .
$$

Then if $\operatorname{rk}\left(\theta^{\prime}\right)>\operatorname{rk}(\xi)$, we have $\rho_{\Theta}^{2} \leqslant \rho_{\Xi}^{2}$. This proves the theorem if hypothesis (H1) holds.

Next, suppose that hypothesis (H2) holds, and write $\xi=(r, \mu, \Delta)$. View $\Delta$ as variable, and consider the quadratic equation in $\Delta$

$$
\rho_{\Xi}^{2}=\frac{r^{2}}{2(r+1)} \Delta
$$


The Ample CONe of MOduli SPACES OF SheAVES ON The Plane

TABLE 3. Computation of $\Delta_{0}(r, \mu), \Delta_{1}(r, \mu)$, and $x^{+}(r, \mu)$

\begin{tabular}{cccccccccccccccc}
\hline$r$ & $\mu$ & $\Delta_{0}$ & $\Delta_{1}$ & $x^{+}$ & $r$ & $\mu$ & $\Delta_{0}$ & $\Delta_{1}$ & $x^{+}$ & $r$ & $\mu$ & $\Delta_{0}$ & $\Delta_{1}$ & $x^{+}$ \\
\hline 1 & 1 & 2 & 1.00 & 0 & 4 & $\frac{1}{2}$ & $\frac{7}{8}$ & 0.81 & 0 & 5 & 1 & $\frac{6}{5}$ & 1.13 & 0.46 \\
2 & $\frac{1}{2}$ & $\frac{7}{8}$ & 0.25 & 0 & 4 & $\frac{3}{4}$ & $\frac{29}{32}$ & 0.67 & 0.53 & 6 & $\frac{1}{6}$ & $\frac{67}{72}$ & 0.03 & 0 \\
2 & 1 & $\frac{3}{2}$ & 1.11 & 0.30 & 4 & 1 & $\frac{5}{4}$ & 1.13 & 0.44 & & 6 & $\frac{1}{3}$ & $\frac{8}{9}$ & 0.79 & 0 \\
3 & $\frac{1}{3}$ & $\frac{8}{9}$ & 0.11 & 0 & 5 & $\frac{1}{5}$ & $\frac{23}{25}$ & 0.04 & 0 & & 6 & $\frac{1}{2}$ & $\frac{17}{24}$ & 0.64 & 0.17 \\
3 & $\frac{2}{3}$ & $\frac{8}{9}$ & 0.57 & 0.37 & 5 & $\frac{2}{5}$ & $\frac{17}{25}$ & 0.65 & 0 & 6 & $\frac{2}{3}$ & $\frac{13}{18}$ & 0.58 & 0.46 \\
3 & 1 & $\frac{4}{3}$ & 1.13 & 0.39 & 5 & $\frac{3}{5}$ & $\frac{17}{25}$ & 0.48 & 0.37 & 6 & $\frac{5}{6}$ & $\frac{67}{72}$ & 0.78 & 0.68 \\
4 & $\frac{1}{4}$ & $\frac{29}{32}$ & 0.06 & 0 & 5 & $\frac{4}{5}$ & $\frac{23}{25}$ & 0.73 & 0.62 & 6 & 1 & $\frac{7}{6}$ & 1.13 & 0.48 \\
\hline
\end{tabular}

The coefficients of this equation depend only on $r$ and $\mu$. Assuming that this equation has roots, let $\Delta_{1}(r, \mu)$ be the greater of the two roots. Then the theorem is true for $\xi$ if $\Delta \geqslant \Delta_{1}(r, \mu)$. Let $\Delta_{0}(r, \mu)$ be the minimal discriminant of a rank $r$ and slope $\mu$ sheaf satisfying hypothesis (H2). We record the values of $\Delta_{0}(r, \mu)$ and $\Delta_{1}(r, \mu)$ for all pairs $(r, \mu)$ with $1 \leqslant r \leqslant 6$ and $0<\mu \leqslant 1$ in Table 3. For later use, we also record the value of the right endpoint $\left(x^{+}(r, \mu), 0\right)$ of the wall $W(\Xi)$ corresponding to the character $\left(r, \mu, \Delta_{0}\right)$.

In every case, we find $\Delta_{0}(r, \mu) \geqslant \Delta_{1}(r, \mu)$, as required. We note that $\Delta_{1}\left(6, \frac{1}{3}\right)>\frac{13}{18}$, so the proof does not apply to $\xi=\left(6, \frac{1}{3}, \frac{13}{18}\right)$.

When $\xi=\left(6, \frac{1}{3}, \frac{13}{18}\right)$, we put $\Xi=\left((5,0,0),\left(6, \frac{1}{3}, \frac{13}{18}\right),(1,2,6)\right)$ and compute $\rho_{\Xi}^{2}=4$. If $\operatorname{rk}\left(\theta^{\prime}\right)>6$, then Proposition 8.3 gives $\rho_{\Theta}^{2} \leqslant \frac{13}{7}$, so $W(\Xi)$ is not nested in $W(\Theta)$ in this case either.

The proof of the theorem also gives the following nonemptiness result.

Corollary 8.4. If hypothesis ( $\mathrm{H} 1)$ or $(\mathrm{H} 2)$ holds, then $W(\Xi)$ is nonempty. If $\xi=\left(6, \frac{1}{3}, \frac{13}{18}\right)$, the wall corresponding to $\xi^{\prime}=(5,0,0)$ is nonempty.

\subsection{The ample cone, large-discriminant case}

Here we finish the proof that $W(\Xi)$ is the Gieseker wall if $\xi$ satisfies hypothesis (H1). View $\xi=\xi(\Delta)=(\operatorname{rk}(\xi), \mu(\xi), \Delta)$ as having fixed rank and slope and variable $\Delta$, so that the extremal triple $\Xi=\Xi(\Delta)$ decomposing $\xi(\Delta)$ depends on $\Delta$. We begin with the following lemma that will also be useful in the small-rank case (H2).

Lemma 8.5. The right endpoint $x_{\Xi(\Delta)}^{+}=s_{\Xi(\Delta)}+\rho_{\Xi(\Delta)}$ of $W(\Xi(\Delta))$ is a strictly increasing function of $\Delta$, and

$$
\lim _{\Delta \rightarrow \infty} x_{\Xi(\Delta)}^{+}=\mu\left(\xi^{\prime}\right)
$$

Proof. The statement that the function is increasing follows as in the second paragraph of Remark 4.2. The walls $W(\Xi(\Delta))$ are all potential walls for the Chern character $\xi^{\prime}$, so they form a nested family of semicircles foliating the quadrant left of the vertical wall $s=\mu\left(\xi^{\prime}\right)$. If the radius of such a wall is arbitrarily large, then its right endpoint is arbitrarily close to the vertical wall. We saw in the proof of Theorem 8.2 that if $\Delta$ is arbitrarily large, then the radius of $W(\Xi(\Delta))$ is arbitrarily large. 


\section{Coskun And J. Huizenga}

Theorem 8.6. Suppose that $\xi$ satisfies hypothesis (H1), and let $\Xi$ be the extremal triple decomposing $\xi$. Then $W(\Xi)=W_{\max }$, and the primary edge of the ample cone of $M(\xi)$ corresponds to $W(\Xi)$.

Proof. Let $\Delta(\xi)$ be large enough that there is an extremal $\Xi$ that gives curves. Also assume that $\Delta(\xi)$ is large enough that Theorem 8.2 holds. If necessary, further increase $\Delta(\xi)$ so that no rational numbers with denominator at most $\operatorname{rk}(\xi)$ lie in the interval $\left[x_{\Xi}^{+}, \mu\left(\xi^{\prime}\right)\right)$.

Suppose that the decomposition $\Theta$ corresponds to an actual wall $W(\Theta)$ which is at least as large as $W(\Xi)$, and let

$$
0 \rightarrow F \rightarrow E \rightarrow Q \rightarrow 0
$$

be a destabilizing sequence along $W(\Theta)$. Since $F$ is an element of $\mathcal{Q}_{s}$ along $W(\Xi)$, we have $\mu(F) \geqslant x_{\Xi}^{+}$. Now $\operatorname{rk}(F) \leqslant \operatorname{rk}(\xi)$, so by the choice of $\Delta(\xi)$ and the slope-closeness condition (E1) we conclude $\mu(F)=\mu\left(\xi^{\prime}\right)$.

Furthermore, $F$ is $\mu$-semistable. If it were not, by Lemma 8.1 the only possibility would be that $F$ has a subsheaf of slope $\mu(\xi)$. Then $F$ must have a Harder-Narasimhan factor of slope less than $\mu\left(\xi^{\prime}\right)$, and this violates the fact that $F \in \mathcal{Q}_{s}$ for all $s$ along $W(\Theta)$ by our choice of $\Delta(\xi)$.

Finally, the $\mu$-semistability of $F$ implies $\Delta(F) \geqslant \Delta\left(\xi^{\prime}\right)$ by the discriminant-minimality condition (E2). If $\Delta(F)>\Delta\left(\xi^{\prime}\right)$, then $W(\Theta)$ is nested inside $W(\Xi)$. We conclude $\Delta(F)=\Delta\left(\xi^{\prime}\right)$, and $W(\Theta)=W(\Xi)$. Therefore $W(\Xi)$ is the Gieseker wall.

Remark 8.7. The lower bound on $\Delta$ needed for our proof of Theorem 8.6 can be made explicit. We have increased $\Delta$ on several occasions throughout the paper. If $r$ and $\mu$ are fixed, then $\Delta$ needs to be large enough that the following statements hold:

(i) The character $\xi^{\prime \prime}$ is stable (Lemma 4.3).

(ii) The triple $\Xi$ gives curves. Alternately, it is enough to know that if $F \in M\left(\xi^{\prime}\right)$ and $Q \in M\left(\xi^{\prime \prime}\right)$ are general, then $\operatorname{Hom}(F, Q)=0$. The proof of Theorem 7.5 allows us to give a lower bound for $\Delta$ if $\operatorname{hom}(F, Q)$ can be computed for some extremal triple $\Xi$ decomposing a character $\xi$ with rank $r$ and slope $\mu$.

(iii) The wall $W(\Xi)$ is large enough to imply that the destabilizing subobject along $W_{\max }$ has rank at most $r$ (Proposition 8.3).

(iv) The right endpoint $x_{\Xi}^{+}$of $W(\Xi)$ is close enough to $\mu\left(\xi^{\prime}\right)$ that every rational number in $\left[x_{\Xi}^{+}, \mu\left(\xi^{\prime}\right)\right)$ has denominator greater than $r$.

Remark 8.8. As an application of Theorem 8.6 and the discussion in the preceding remark, we explain how our results recover Yoshioka's computation [Yos03] of the ample cone of $M(\xi)$ in the case $c_{1}(\xi)=1$ and $r \geqslant 2$. Let $\theta^{\prime}=(2,0,0)$ and $\theta=(r, 1 / r, P(-1 / r)+(1 / r))$, and let $\Theta$ be the corresponding admissible triple. By Proposition 7.7, the triple $\Theta$ is complete and gives curves. Any triple $\Lambda \succeq \Theta$ also gives curves by Lemma 7.4.

Now, suppose that $\xi$ has positive height and $c_{1}(\xi)=1$. Then either $\xi=\theta$ or $\xi$ is an elementary modification of $\theta$. Let $\Xi$ be the extremal triple decomposing $\xi$. If $W(\Xi)$ is the Gieseker wall, then the curves in $M(\xi)$ constructed in the previous paragraph are orthogonal to the divisor class on $M(\xi)$ coming from $W(\Xi)$, so we only need to check that $W(\Xi)$ is the Gieseker wall. To do this, we verify that if $\Delta \geqslant P(-1 / r)+1 / r$, then statements (i), (iii), and (iv) in Remark 8.7 hold. It is clear that $\xi^{\prime \prime}$ is stable, so statement (i) holds.

To check statements (iii) and (iv), it is enough to verify that they hold for the decomposi- 
tion $\Theta$. For statement (iii), by Proposition 8.3 we must show

$$
\left(r-\frac{1}{2}\right)^{2}=\rho_{\Theta}^{2} \geqslant \frac{r^{2}}{2(r+1)} \Delta(\theta)=\frac{2 r^{2}-r+1}{4 r+4},
$$

which is clear for $r \geqslant 2$. For statement (iv), we need $x_{\Theta}^{+}>-1 / r$; in fact, $x_{\Theta}^{+}=0$ holds.

\subsection{The ample cone, small-rank case}

We next compute the Gieseker wall in the small-rank case.

TheOREM 8.9. Suppose that $\xi$ satisfies hypothesis (H2), and let $\Xi$ be the extremal triple decomposing $\xi$. Then $W(\Xi)=W_{\max }$, and the primary edge of the ample cone of $M(\xi)$ corresponds to $W(\Xi)$.

Proof. We may assume $0<\mu(\xi) \leqslant 1$. Suppose that $\Theta=\left(\theta^{\prime}, \theta, \theta^{\prime \prime}\right)$ is a decomposition of $\xi$ corresponding to an actual wall $W(\Theta)$ which is larger than $W(\Xi)$. Let $F \rightarrow E$ be a destabilizing inclusion corresponding to $W(\Theta)$. We will show $\mu\left(\theta^{\prime}\right)>\mu\left(\xi^{\prime}\right)$. Combining this with Lemma 8.1, Theorem 8.2, and slope closeness (E1) then gives a contradiction.

To prove $\mu\left(\theta^{\prime}\right)>\mu\left(\xi^{\prime}\right)$, we first derive two auxiliary inequalities. We will make use of the nondegenerate symmetric bilinear form $(\xi, \zeta)=\chi(\xi \otimes \zeta)$ on $K\left(\mathbb{P}^{2}\right)$. Let $\gamma$ be a Chern character of positive rank such that $\gamma^{\perp}=\left\langle\xi^{\prime}, \xi\right\rangle$.

First inequality: Since $\mu\left(\theta^{\prime}\right)<\mu(\xi)$, the assumption that $W(\Theta)$ is larger than $W(\Xi)$ means $\left(\theta^{\prime}, \gamma\right)>0$. Indeed, $W(\Theta)=W(\Xi)$ if and only if $\theta^{\prime} \in \gamma^{\perp}$. If $\Delta\left(\theta^{\prime}\right)$ is decreased starting from a character on $\gamma^{\perp}$, then $\left(\theta^{\prime}, \gamma\right)$ increases and the wall $W(\Theta)$ becomes larger.

Second inequality: Put $\zeta_{1}=\operatorname{ch} \mathcal{O}_{\mathbb{P}^{2}}(-1)$ and $\zeta_{2}=\operatorname{ch} \mathcal{O}_{\mathbb{P}^{2}}(-3)$. We observe that $\xi^{\prime}$ lies in either $\zeta_{1}^{\perp}$ or $\zeta_{2}^{\perp}$. Let $i$ be such that $\xi^{\prime} \in \zeta_{i}^{\perp}$; we will show $\left(\theta^{\prime}, \zeta_{i}\right) \leqslant 0$ in either case.

Case 1: $\left(\xi^{\prime}, \zeta_{1}\right)=0$. If $\left(\theta^{\prime}, \zeta_{1}\right)>0$, then $\chi\left(\mathcal{O}_{\mathbb{P}^{2}}(1), F\right)>0$. Suppose $\operatorname{Ext}^{2}\left(\mathcal{O}_{\mathbb{P}^{2}}(1), F\right)=0$. Then there is a nonzero homomorphism $\mathcal{O}_{\mathbb{P}^{2}}(1) \rightarrow F$, and composing with the inclusion $F \rightarrow E$ gives a nonzero homomorphism $\mathcal{O}_{\mathbb{P}^{2}}(1) \rightarrow E$. Since $\xi$ has slope at most 1 and positive height, this contradicts the semistability of $E$.

It remains to prove $\operatorname{Ext}^{2}\left(\mathcal{O}_{\mathbb{P}^{2}}(1), F\right)=0$. Dually, we must show $\operatorname{Hom}\left(F, \mathcal{O}_{\mathbb{P}^{2}}(-2)\right)=0$. If $x_{\Xi}^{+} \geqslant-2$, then since $W(\Theta)$ is larger than $W(\Xi)$, we will have $F \in \mathcal{Q}_{-2}$, proving this vanishing. By Lemma 8.5, we only have to check this inequality when $\Delta(\xi)$ is minimal subject to satisfying hypothesis (H2). We carried out this computation in Table 3.

Case 2: $\left(\xi^{\prime}, \zeta_{2}\right)=0$. If $\left(\theta^{\prime}, \zeta_{2}\right)>0$, then either $\operatorname{Hom}\left(\mathcal{O}_{\mathbb{P}^{2}}(3), F\right) \operatorname{or}_{\operatorname{Ext}^{2}}\left(\mathcal{O}_{\mathbb{P}^{2}}(3), F\right)=$ $\operatorname{Hom}\left(F, \mathcal{O}_{\mathbb{P}^{2}}\right)^{*}$ is nonzero. Clearly $\operatorname{Hom}\left(\mathcal{O}_{\mathbb{P}^{2}}(3), F\right)=0$ by Lemma 8.1. We must therefore show $\operatorname{Hom}\left(F, \mathcal{O}_{\mathbb{P}^{2}}\right)=0$. This follows from $x_{\Xi}^{+} \geqslant 0$, which is again true.

Now we use the inequalities $\left(\theta^{\prime}, \gamma\right)>0$ and $\left(\theta^{\prime}, \zeta_{i}\right) \leqslant 0$ to prove $\mu\left(\theta^{\prime}\right)>\mu\left(\xi^{\prime}\right)$. There is a character $\nu$ such that if $\eta$ has positive rank, then $(\eta, \nu) \geqslant 0$ (respectively, $(\eta, \nu)>0$ ) if and only if $\mu(\eta) \geqslant \mu\left(\xi^{\prime}\right)$ (respectively, $\mu(\eta)>\mu\left(\xi^{\prime}\right)$ ). We summarize the known information about the signs of various pairs of characters here, noting that $\left(\xi, \zeta_{i}\right)<0$ since $\xi$ has positive height.

\begin{tabular}{c|ccc}
$(-,-)$ & $\gamma$ & $\zeta_{i}$ & $\nu$ \\
\hline$\xi$ & 0 & $<0$ & $>0$ \\
$\xi^{\prime}$ & 0 & 0 & 0 \\
$\theta^{\prime}$ & $>0$ & $\leqslant 0$ &
\end{tabular}

The character $\nu$ is in $\left(\xi^{\prime}\right)^{\perp}$, and $\gamma$ and $\zeta_{i}$ form a basis for $\left(\xi^{\prime}\right)^{\perp}$. Write $\nu=a \gamma+b \zeta_{i}$ as a linear combination. Since $0<(\xi, \nu)=b\left(\xi, \zeta_{i}\right)$, we find $b<0$. The character $\nu$ has rank 0 , so this forces 


\section{Coskun And J. Huizenga}

$a>0$. We conclude

$$
\left(\theta^{\prime}, \nu\right)=a\left(\theta^{\prime}, \gamma\right)+b\left(\theta^{\prime}, \zeta_{i}\right)>0
$$

so $\mu\left(\theta^{\prime}\right)>\mu\left(\xi^{\prime}\right)$.

We finish the paper by considering the last remaining case.

Theorem 8.10. Let $\xi=\left(6, \frac{1}{3}, \frac{13}{18}\right)$, and let $\Xi$ be the decomposition of $\xi$ with $\xi^{\prime}=(5,0,0)$. Then $W(\Xi)=W_{\max }$, and the primary edge of the ample cone corresponds to $W(\Xi)$.

Proof. We use the same notation as in the proof of the previous theorem. Through a computation, we see $x_{\Xi}^{+}=0$, so since $W(\Theta)$ is larger than $W(\Xi)$, we have $F \in \mathcal{Q}_{\epsilon}$ for some small $\epsilon>0$. Consider the Harder-Narasimhan filtration

$$
0 \subset F_{1} \subset \cdots \subset F_{\ell}=F .
$$

Every quotient $\operatorname{gr}_{i}$ of this filtration satisfies $0<\mu\left(\mathrm{gr}_{i}\right) \leqslant \frac{1}{3}$. Since $\operatorname{gr}_{i}$ is semistable, we find $\chi\left(\operatorname{gr}_{i}, \mathcal{O}_{\mathbb{P}^{2}}\right) \leqslant 0$ for all $i$. It follows that $\chi\left(\theta^{\prime}, \operatorname{ch}\left(\mathcal{O}_{\mathbb{P}^{2}}\right)\right) \leqslant 0$ as well. A straightforward computation using this inequality and $\chi\left(\theta^{\prime}, \gamma\right)>0$ shows $\mu\left(\theta^{\prime}\right)>\frac{2}{7}$. This contradicts Theorem 8.2 since every rational number in the interval $\left(\frac{2}{7}, \frac{1}{3}\right)$ has denominator greater than 6 .

\section{ACKNOWLEDGEMENTS}

We are grateful to Daniele Arcara, Arend Bayer, Aaron Bertram, Lawrence Ein, Joe Harris, Brendan Hassett, Emanuele Macrì, and Matthew Woolf for many useful conversations about the geometry of moduli spaces of semistable sheaves. We thank the referee for many useful comments and improvements.

\section{REFERENCES}

AB13 D. Arcara and A. Bertram, Bridgeland-stable moduli spaces for $K$-trivial surfaces (with an appendix by Max Lieblich, J. Eur. Math. Soc. (JEMS) 15 (2013), no. 1, 1-38; http://dx.doi. org/10.4171/JEMS/354.

ABCH13 D. Arcara, A. Bertram, I. Coskun, and J. Huizenga, The minimal model program for the Hilbert scheme of points on $\mathbb{P}^{2}$ and Bridgeland stability, Adv. Math. 235 (2013), 580-626; http: //dx.doi.org/10.1016/j.aim.2012.11.018.

BC13 A. Bertram and I. Coskun, The birational geometry of the Hilbert scheme of points on surfaces, in Birational Geometry, Rational Curves, and Arithmetic (Springer, New York, 2013), 15-55; http://dx.doi.org/10.1007/978-1-4614-6482-2_2.

BM11 A. Bayer and E. Macrì, The space of stability conditions on the local projective plane, Duke Math. J. 160 (2011), no. 2, 263-322; http://dx.doi.org/10.1215/00127094-1444249.

BM14a _ MMP for moduli of sheaves on K3s via wall-crossing: nef and movable cones, Lagrangian fibrations, Invent. Math. 198 (2014), no. 3, 505-590; http://dx.doi.org/10.1007/ s00222-014-0501-8.

BM14b_, Projectivity and birational geometry of Bridgeland moduli spaces, J. Amer. Math. Soc. 27 (2014), no. 3, 707-752; http://dx.doi.org/10.1090/S0894-0347-2014-00790-6.

Bri07 T. Bridgeland, Stability conditions on triangulated categories, Ann. of Math. (2) 166 (2007), no. 2, 317-345; http://dx.doi.org/10.4007/annals.2007.166.317.

Bri08_ Stability conditions on K3 surfaces, Duke Math. J. 141 (2008), no. 2, 241-291; http: //dx.doi.org/10.1215/S0012-7094-08-14122-5. 


\section{ThE AMPLE CONE OF MODUli SPACES OF SHEAVES ON THE PLANE}

CH14 I. Coskun and J. Huizenga, Interpolation, Bridgeland stability and monomial schemes in the plane, J. Math. Pures Appl. (9) 102 (2014), no. 5, 930-971; http://dx.doi.org/10.1016/j. matpur.2014.02.010.

CHW15 I. Coskun, J. Huizenga, and M. Woolf, The effective cone of the moduli spaces of sheaves on the plane, J. Eur. Math. Soc. (JEMS), to appear, arXiv:1401.1613.

DLP85 J.-M. Drezet and J. Le Potier, Fibrés stables et fibrés exceptionnels sur $\mathbf{P}_{2}$, Ann. Sci. École Norm. Sup. (4) 18 (1985), no. 2, 193-243; http://www.numdam.org/item?id=ASENS_1985_4_ 18_2_193_0.

Dre86 J.-M. Drezet, Fibrés exceptionnels et suite spectrale de Beilinson généralisée sur $\mathbf{P}_{2}(\mathbf{C})$, Math. Ann. 275 (1986), no. 1, 25-48; http://dx.doi.org/10.1007/BF01458581.

HL10 D. Huybrechts and M. Lehn, The geometry of moduli spaces of sheaves, second edn., Cambridge Math. Lib. (Cambridge Univ. Press, Cambridge, 2010); http://dx.doi.org/10.1017/ CB09780511711985.

Hui15 J. Huizenga, Effective divisors on the Hilbert scheme of points in the plane and interpolation for stable bundles, J. Algebraic Geom., electronically published on 29 July 2015, http://dx. doi.org/10.1090/jag/652, to appear in print.

Laz04 R. Lazarsfeld, Positivity in algebraic geometry. I. classical setting: line bundles and linear series, Ergeb. Math. Grenzgeb. (3) Folge, vol. 48 (Springer-Verlag, Berlin, 2004).

Li93 J. Li, Algebraic geometric interpretation of Donaldson's polynomial invariants, J. Differential Geom. 37 (1993), no. 2, 417-466; http://projecteuclid.org/euclid.jdg/1214453683.

LP97 J. Le Potier, Lectures on vector bundles, Cambridge Stud. Adv. Math., vol. 54 (Cambridge Univ. Press, Cambridge, 1997).

LQZ03 W.-P. Li, Z. Qin, and Q. Zhang, Curves in the Hilbert schemes of points on surfaces, Vector Bundles and Representation Theory (Columbia, MO, 2002), Contemp. Math., vol. 322 (Amer. Math. Soc. Providence, RI, 2003), 89-96; http://dx.doi.org/10.1090/conm/322/05681.

LZ13 C. Li and X. Zhao, The MMP for deformations of Hilbert schemes of points on the projective plane, arXiv:1312.1748.

MM13 A. Maciocia and C. Meachan, Rank 1 Bridgeland stable moduli spaces on a principally polarized abelian surface, Int. Math. Res. Not. IMRN 2013 (2013), no. 9, 2054-2077; http://dx.doi. org/10.1093/imrn/rns107.

MYY11 H. Minamide, S. Yanagida, and K. Yoshioka, Fourier-Mukai transforms and the wall-crossing behavior for Bridgeland's stability conditions, arXiv:1106.5217.

MYY14 Some moduli spaces of Bridgeland's stability conditions, Int. Math. Res. Not. IMRN 2014 (2014), no. 19, 5264-5327; http://dx.doi.org/10.1093/imrn/rnt126.

Nue14 H. Nuer, Projectivity and birational geometry of moduli spaces of Bridgeland stable objects on an Enriques surface, arXiv:1406.0908.

Ohk10 R. Ohkawa, Moduli of Bridgeland semistable objects on $\mathbf{P}^{2}$, Kodai Math. J. 33 (2010), no. 2, 329-366; http://dx.doi.org/10.2996/kmj/1278076346.

Str84 S. A. Strømme, Ample divisors on fine moduli spaces on the projective plane, Math. Z. 187 (1984), no. 3, 405-423; http://dx.doi.org/10.1007/BF01161956.

Woo13 M. Woolf, Nef and effective cones on the moduli space of torsion sheaves on the projective plane, arXiv:1305.1465.

Yos03 _ A note on moduli of vector bundles on rational surfaces, J. Math. Kyoto Univ. 43 (2003), no. 1, 139-163; http://projecteuclid.org/euclid.kjm/1250283744.

Yos12 K. Yoshioka, Bridgeland's stability and the positive cone of the moduli spaces of stable objects on an abelian surface, arXiv:1206.4838.

YY14 S. Yanagida and K. Yoshioka, Bridgeland's stabilities on abelian surfaces, Math. Z. 276 (2014), no. 1-2, 571-610; http://dx.doi.org/10.1007/s00209-013-1214-1. 


\section{Coskun And J. Huizenga}

Izzet Coskun coskun@math.uic.edu

Department of Mathematics, Statistics and CS, University of Illinois at Chicago, Chicago, IL 60607, USA

Jack Huizenga huizenga@math.uic.edu

Department of Mathematics, Statistics and CS, University of Illinois at Chicago, Chicago, IL 60607, USA 